\title{
Supersoft X-rays reveal a classical nova in the M 31 globular cluster Bol 126
}

\author{
M. Henze ${ }^{1}$, W. Pietsch ${ }^{1}$, F. Haberl ${ }^{1}$, M. Della Valle ${ }^{2,3}$, A. Riffeser ${ }^{4}$, G. Sala ${ }^{5,6}$, D. Hatzidimitriou ${ }^{7,8}$, F. Hofmann ${ }^{1}$, \\ D. H. Hartmann ${ }^{9}$, J. Koppenhoefer ${ }^{1,4}$, S. Seitz ${ }^{1,4}$, G. G. Williams ${ }^{10}$, K. Hornoch ${ }^{11}$, K. Itagaki ${ }^{12}$, F. Kabashima ${ }^{13}$, \\ K. Nishiyama ${ }^{13}$, G. Xing ${ }^{14}$, C. H. Lee ${ }^{15}$, E. Magnier ${ }^{16}$, and K. Chambers ${ }^{16}$ \\ 1 Max-Planck-Institut für extraterrestrische Physik, Postfach 1312, Giessenbachstr., 85741 Garching, Germany \\ e-mail: mhenze@mpe.mpg.de \\ 2 INAF-Napoli, Osservatorio Astronomico di Capodimonte, Salita Moiariello 16, 80131 Napoli, Italy \\ 3 International Centre for Relativistic Astrophysics, Piazzale della Repubblica 2, 65122 Pescara, Italy \\ ${ }^{4}$ University Observatory Munich, Scheinerstrasse 1, 81679 München, Germany \\ 5 Departament de Física i Enginyeria Nuclear, EUETIB, Universitat Politècnica de Catalunya, c/Comte d'Urgell 187, \\ 08036 Barcelona, Spain \\ 6 Institut d'Estudis Espacials de Catalunya, c/Gran Capità 2-4, Ed. Nexus-201, 08034 Barcelona, Spain \\ 7 Department of Astrophysics, Astronomy and Mechanics, Faculty of Physics, University of Athens, Panepistimiopolis, \\ GR15784 Zografos, Athens, Greece \\ 8 Foundation for Research and Technology Hellas, IESL, Greece \\ 9 Department of Physics and Astronomy, Clemson University, Clemson, SC 29634-0978, USA \\ 10 Steward Observatory, 933 North Cherry Avenue, Tucson, AZ 85721, USA \\ 11 Astronomical Institute, Academy of Sciences, CZ-251 65 Ondřejov, Czech Republic \\ 12 Itagaki Astronomical Observatory, Teppo-cho, Yamagata 990-2492, Japan \\ 13 Miyaki-Argenteus Observatory, Miyaki-cho, Saga-ken, Japan \\ 14 Xingming Observatory, Mt. Nanshan, Urumqi, Xinjiang, PR China \\ 15 Graduate Institute of Astronomy, National Central University, Jhongli 32001, Taiwan \\ ${ }^{16}$ Institute for Astronomy, University of Hawaii at Manoa, Honolulu, HI 96822, USA
}

Received 9 August 2012 / Accepted 2 November 2012

\section{ABSTRACT}

Context. Classical novae $(\mathrm{CNe})$ represent the main class of supersoft X-ray sources (SSSs) in the central region of our neighbouring galaxy M 31. Only three confirmed novae and three SSSs have been discovered in globular clusters (GCs) of any galaxy so far, of which one nova and two SSSs (including the nova) were found in M 31 GCs.

Aims. To study the SSS state of CNe we carried out a high-cadence X-ray monitoring of the M 31 central area with XMM-Newton and Chandra. This project is supplemented by regular optical monitoring programmes at various observatories.

Methods. We analysed X-ray and optical monitoring data of a new transient X-ray source in the M 31 GC Bol 126, discovered serendipitously in Swift observations. Our optical data set was based on regular M 31 monitoring programmes from five different small telescopes and was reduced using a homogeneous method. Additionally, we made use of Pan-STARRS 1 data obtained during the PAndromeda survey. We extracted light curves of the source in the optical and X-rays, as well as X-ray spectra.

Results. Our observations reveal that the X-ray source in Bol 126 is the third SSS in an M 31 GC and can be confirmed as the second CN in the M 31 GC system. This nova is named M31N 2010-10f. Its properties in the X-ray (high black-body temperature, short SSS phase) and optical (relatively high maximum magnitude, fast decline) regimes agree with a massive white $\operatorname{dwarf}\left(M_{\mathrm{WD}} \gtrsim 1.3 M_{\odot}\right)$ in the binary system. Incorporating the data on previously found (suspected) novae in M $31 \mathrm{GCs}$ we used our high-cadence X-ray monitoring observations to estimate a tentative nova rate in the $\mathrm{M} 31 \mathrm{GC}$ system of $0.05 \mathrm{yr}^{-1} \mathrm{GC}^{-1}$. An optical estimate, based on the recent 10.5-year WeCAPP survey, gives a lower nova rate, which is compatible with the X-ray rate on the $95 \%$ confidence level. Conclusions. Although still based on small-number statistics, there is growing evidence that the nova rate in GCs is higher than expected from primordial binary formation and under conditions as in the field. Dynamical binary formation and/or additional accretion from the intracluster medium are possible scenarios for an increased nova rate, but observational confirmation for this enhancement has been absent, so far. Regular X-ray monitoring observations of M 31 provide a promising strategy to find these novae.

Key words. galaxies: individual: M 31 - novae, cataclysmic variables - X-rays: binaries - stars: individual: M31N 2010-10f globular clusters: individual: Bol 126

* Partly based on observations with XMM-Newton, an ESA Science Mission with instruments and contributions directly funded by ESA Member States and NASA.

$\star \star$ Tables 1-3 are available in electronic form at

http://www . aanda.org

\section{Introduction}

Classical novae $(\mathrm{CNe})$, a subtype of cataclysmic variables $(\mathrm{CVs})$ showing luminous optical outbursts (see e.g. Bode \& Evans 2008), are rarely detected in globular clusters (GCs). Only three such discoveries are known: in the Galactic GC M 80 (nova T Sco; Luther 1860; Dieball et al. 2010), in a GC of the 
elliptical galaxy M 87 (Shara et al. 2004), and in the GC Bol 111 of our large neighbour galaxy M 31 (Shafter \& Quimby 2007; Henze et al. 2009, hereafter SQ2007, HPH2009). Shara et al. (2004) argued that a fourth candidate, nova 1938 in the Galactic GC M 14, was not a genuine GC nova.

An equally rare event is the discovery of a supersoft X-ray source (SSS; Trümper et al. 1991; Greiner et al. 1991) in a GC; only three such objects have been found to date. The first was the transient SSS 1E 1339.8+2837 in the Galactic GC M3 (NGC 5272; Verbunt et al. 1995; Dotani et al. 1999). This source was subsequently identified as a CV with unusual features (Edmonds et al. 2004; Stacey et al. 2011) and might not fit into the classical picture of SSSs as nuclear burning white dwarfs (WDs; see Kahabka \& van den Heuvel 1997, and references therein). The two other SSSs have been reported in M 31 GCs (HPH2009).

Interestingly, one of these two M 31 SSSs was identified by HPH2009 with the GC nova discovered by SQ2007. Classical novae have been found to constitute the majority of SSSs in the central region of M 31 (Pietsch et al. 2005a). Recently, Henze et al. (2011) published a catalogue of 60 novae in M 31 with a soft X-ray counterpart, a number significantly higher than for any other galaxy, including the Milky Way $(<30$; see Schwarz et al. 2011).

The SSS emission in $\mathrm{CNe}$ is believed to be a signature of stable hydrogen burning in accreted material on the surface of the WD that is not ejected during the nova outburst (Starrfield et al. 1974; Sala \& Hernanz 2005). Nova models describe that hydrogen-rich, degenerate matter accumulates on the WD surface until a thermonuclear runaway leads to a violent ejection of the hot envelope (e.g. Starrfield 1989). This causes a strong rise in optical luminosity (on average 9-12 mag) within time scales of hours to days: the optical nova outburst. The underlying SSS becomes observable when the expansion of the ejected envelope reduces its opacity sufficiently (Krautter 2002). This time scale is defined here as the SSS turn-on time, in agreement with earlier papers and theoretical work (e.g. Hachisu \& Kato 2006, 2010), and should not be confused with the onset of the stable nuclear burning shortly after the outburst. As soon as its hydrogen fuel is exhausted, the SSS disappears. This time scale, the SSS turn-off time, mainly depends on the amount of hydrogen left on the WD surface after the outburst (Sala \& Hernanz 2005). For massive WDs, the expected SSS duration is very short (<100 d; Tuchman \& Truran 1998; Sala \& Hernanz 2005; Hachisu \& Kato 2010).

It seems surprising that among the more than 900 nova candidates known in M 31 to date ${ }^{1}$ there is only a single GC nova. However, this might be explained by the fact that almost all optical surveys for CNe in M 31 that were conducted in the past (see Shafter \& Irby 2001; Henze et al. 2008, and references therein) searched for suddenly appearing objects that were not visible before and fade back to invisibility in days to weeks. This condition is certainly not fulfilled by $\mathrm{CNe}$ in relatively bright GCs, where the optical background light of the GC itself makes a photometric discovery of a nova outburst much more complicated.

The first search specifically for novae in (54) M 31 GCs was carried out by Ciardullo et al. (1990b) based on $\mathrm{H} \alpha$ data obtained by Ciardullo et al. (1987, 1990a). Another pioneering work was the first, and so far only, spectroscopic survey by Tomaney et al. (1992), who monitored more than 200 M 31 GCs over an effective survey time of one year. Both studies did not detect any

\footnotetext{
1 August 2012: see the MPE online catalogue at

http://www . mpe.mpg. de/ m31novae/opt/m31/index . php
}

nova eruptions and reported upper limits on the nova rate in the M 31 GC system that were below the tentative rate later found by HPH2009 from their X-ray data.

This circumstance led HPH2009 to note that "the detection of supersoft emission from a hydrogen-burning post-nova atmosphere is not affected [by the light of the GC]" and to speculate that "the connection of CNe to SSSs in X-rays provides a useful possibility to detect CNe in GCs". In the present paper, we make use of this connection and describe the second nova found in an M 31 GC, which was first discovered as an SSS. The nova was detected in the GC Bol 126 and given the name M31N 2010-10f. Section 2 provides detailed information on our X-ray and optical data sets. Results are presented in Sect. 3 and discussed in Sect. 4 together with implications on the M 31 GC nova rate.

\section{Observations and data analysis}

\subsection{X-ray observations}

The new X-ray source in the M 31 GC Bol 126 was discovered serendipitously by Pietsch et al. (2010c) during our Swift X-ray telescope (XRT; Burrows et al. 2005) target of opportunity monitoring observations of the recurrent nova M31N 1963-09c (see e.g. Pietsch et al. 2010a,b, and Henze et al., in prep.). Additional Swift observations followed the light curve of the object until the beginning of our regular X-ray monitoring $(\text { Pietsch 2010) })^{2}$ of the M 31 central region with the telescopes XMM-Newton and Chandra (PI: W. Pietsch). This programme used XMM-Newton with the European Photon Imaging Camera (EPIC; Strüder et al. 2001; Turner et al. 2001) as its primary instrument, while Chandra was operated with the HighResolution Camera imaging detector (HRC-I; Murray et al. 2000). In Table 1 we list all X-ray observations.

Data analysis for the Swift XRT was carried out using the source statistics (sosta) tool within the HEAsoft XIMAGE package (version 4.5.1.). This approach included corrections for detector exposure (exposure maps created using the XRT software task xrtexpomap) and the point spread function (PSF) of the source (XIMAGE command psf).

The XMM-Newton data were analysed using the XMMSAS v11.0 software (XMM-Newton Science Analysis System; Gabriel et al. 2004) ${ }^{3}$. Our data analysis techniques differ from the standard processing and are described in detail in Henze et al. (2010b). The Chandra HRC-I observations were reduced with the CIAO v4.4 software package (Chandra Interactive Analysis of Observations; Fruscione et al. 2006) ${ }^{4}$ and with adapted versions of XMMSAS tools, starting with a re-processing of the level 2 event files. With respect to Henze et al. (2010b), our Chandra data reduction procedures have been updated to allow for better treatment of the HRC-I PSF. We here used XMMSAS only to create a background map (tool: esplinemap) and based the source detection solely on the CIAO wavdetect algorithm. For an extensive description of our Chandra analysis pipeline see Hofmann et al. (in prep.). The astrometry for the detected $\mathrm{X}$-ray sources was corrected with respect to the catalogue of Kaaret (2002), which was calibrated astrometrically using the Two Micron All Sky Survey (2MASS, Cutri et al. 2003).

$\mathrm{X}$-ray spectra, extracted from the XMM-Newton observations, were analysed in XSPEC (Arnaud 1996, version 12.7.0). For the resulting spectral model, specific energy conversion factors were estimated in XSPEC using the fakeit command. We

\footnotetext{
http://www.mpe.mpg.de/ m31novae/xray/index.php

3 http://xmm.esac.esa.int/external/xmm_data_analysis/

4 http://cxc.harvard.edu/ciao/
} 
searched for variability within the individual observations by extracting light curves using evselect for XMM-Newton and dmextract for Chandra data. Additionally, Chandra detections were analysed using the glvary tool, which applies the algorithm of Gregory \& Loredo (1992) to classify source variability. A search for light curve periodicities was conducted using the XRONOS tasks of HEASARCs software package FTOOLS ${ }^{5}$ (Blackburn 1995).

\subsection{Optical observations}

Motivated by the discovery of the new X-ray source in an M 31 GC, we re-analysed archival optical observations in search for a counterpart. The data set consisted of observations carried out with small telescopes participating in nova search projects and of M 31 monitoring observations obtained during the Pan-STARRS 1 survey (Panoramic Survey Telescope and Rapid Response System). This allowed for a detailed coverage of the Bol 126 light curve.

\subsubsection{Observations with small telescopes}

Optical data were obtained at five different observatories in the context of regular M 31 monitoring programmes with the following telescopes: (a) the Livermore Optical Transient Imaging System (Super-LOTIS, Williams et al. 2008), a robotic $60 \mathrm{~cm}$ telescope with an E2V CCD $(2 \mathrm{k} \times 2 \mathrm{k})$ located at Steward Observatory, Kitt Peak, Arizona, USA (observer: G.G. Williams); (b) a Meade 200R $40 \mathrm{~cm} \mathrm{f} / 9.8$ reflector, plus SBIG STL1001E camera, at Miyaki-Argenteus Observatory, Japan (observers: F. Kabashima and K. Nishiyama); (c) a $50 \mathrm{~cm}$ f/6 telescope, with BITRAN BN-52E(KAF-1001E) camera, located at Itagaki Astronomical Observatory, Japan (observer: K. Itagaki); (d) a $35 \mathrm{~cm}$ f/7.5 Celestron C14 Schmidt-Cassegrain telescope at Xingming Observatory, China (observer: G. Xing); (e) a $65 \mathrm{~cm}$ telescope, with G2CCD-3200 camera, at Ondřejov Observatory, Czech Republic (observers: K. Hornoch, M. Wolf, P. Hornochová, P. Kušnirák and P. Zasche). While observatories (b)-(d) took unfiltered images, Super-LOTIS used a Johnson $R$ and Ondřejov a Kron-Cousins $R$ filter. Observation dates are given in Table 2.

Images from the first four telescopes were reduced and calibrated in a homogeneous way. This procedure made use of the TERAPIX software packages SExtractor (Bertin \& Arnouts 1996) for source extraction, SWarp (Bertin et al. 2002) for image stacking, and SCAMP (Bertin 2006) for image calibration. Image reduction procedures corrected for the strong background light of M 31 and specific detection thresholds were used to create clean source catalogues. The astrometric and photometric solutions were computed in SCAMP using $R$ magnitudes from the M 31 part of the Local Group Galaxy Survey (LGGS, Massey et al. 2006). Photometric uncertainties were estimated from all sufficiently star-like objects in a 1 mag range around the magnitude of Bol 126.

Data from the Ondřejov Observatory were analysed using the SIMS $^{6}$ and Munipack ${ }^{7}$ programmes. Reduced images of the same series were co-added to improve the signal-to-noise ratio (total exposure time varied from $600 \mathrm{~s}$ up to $1800 \mathrm{~s}$ ). The gradient of the galaxy background of co-added images was flattened by the spatial median filter using SIMS. These processed

\footnotetext{
5 http://heasarc.gsfc.nasa.gov/ftools/

6 http://ccd.mii.cz/

7 http://munipack.astronomy.cz/
}

images were used for aperture photometry, carried out in GAIA ${ }^{8}$. Relative photometry was performed using brighter field stars that were calibrated using standard Landolt fields.

The resulting magnitudes of Bol 126 from both samples agree well (see Table 2 and Sect. 3).

\subsubsection{Observations with Pan-STARRS 1}

The PAndromeda survey was designed to identify gravitational microlensing events towards M 31 within the Pan-STARRs survey (PS1). It monitors M 31 for five months per year and $30 \mathrm{~min}$ per night (including overhead). With the $7 \mathrm{deg}^{2}$ field of the Giga Pixel Camera (GPC) mounted on the $1.8 \mathrm{~m}$ telescope on Haleakala (Maui, US) the entirety of M 31 can be observed with one pointing.

The data taken in the first PAndromeda season as well as the data reduction are described in Lee et al. (2012a). In short, the astrometric accuracy of the final data is of the order of $0 .{ }^{\prime} 1$, when compared to the SDSS-DR7 catalogue which was not used to derive the astrometric solution. The photometric accuracy can be seen in Fig. 10 of Lee et al. (2012a); it is 0.01 mag for a magnitude of 16 in the bulge of M31. Since the colour terms of the $r_{\mathrm{p} 1}$ and $i_{\mathrm{p} 1}$ Pan-STARRS filters relative to the corresponding SDSS filter systems are small, $\left(r_{\mathrm{P} 1}-r_{\mathrm{SDSS}}\right)=0.000-$ $0.007(g-r)_{\text {SDSS }}$ and $\left(i_{\mathrm{P} 1}-i_{\mathrm{SDSS}}\right)=0.004-0.014(g-r)_{\mathrm{SDSS}}$, the Pan-STARRS magnitudes can be considered as equivalent to the SDSS magnitudes for this work.

Light curves for variable objects are derived by difference imaging technique (Alard \& Lupton 1998) using the implementation of Gössl \& Riffeser (2002); Gössl et al. (2004) as described in Lee et al. (2012a). The PAndromeda data were successfully searched for microlensing events (Lee et al. 2012a) and are currently being analysed to study cepheids, eclipsing binaries and novae. The brightest and shortest nova found in PAndromeda data up to now was the one discussed in this paper. Its light curves in the $r_{\mathrm{p} 1}$ and $i_{\mathrm{p} 1}$ filters are given in Table 3 (in AB-magnitudes).

\section{Results}

The position of the new X-ray source was determined from Chandra observation 12110, because the HRC-I detector has the best spatial resolution and the source had the highest count rate in this pointing (see Table 1). The coordinates are $\mathrm{RA}=00^{\mathrm{h}} 42^{\mathrm{m}} 43.70^{\mathrm{s}}$, Dec $=+41^{\circ} 12^{\prime} 43^{\prime \prime}{ }^{\prime} 0(\mathrm{~J} 2000,1 \sigma$ accuracy of 0 ." 8 ), which agree well (distance 0 ." 4 ) with the position of the M 31 GC Bol 126: RA $=00^{\mathrm{h}} 42^{\mathrm{m}} 43.681^{\mathrm{s}}$, Dec $=+41^{\circ} 12^{\prime} 42^{\prime \prime} .70$ according to the Revised Bologna Catalogue of M 31 globular clusters and candidates (RBC; version 4.0, Dec 2009; Galleti et al. 2004). No X-ray source was previously known at this position (e.g. Kaaret 2002; Kong et al. 2002; Williams et al. 2004; Pietsch et al. 2005b; Stiele et al. 2011).

We simultaneously fitted the XMM-Newton EPIC pn spectra (single-pixel events) of the X-ray source using an absorbed black body model with best-fit parameters $k T=74_{-26}^{+30} \mathrm{eV}$ and $N_{\mathrm{H}}=$ $\left(0.4_{-0.4}^{+0.9}\right) \times 10^{21} \mathrm{~cm}^{-2}$, resulting in a formal unabsorbed luminosity of $1.3 \times 10^{36} \mathrm{erg} \mathrm{s}^{-1}$. Therefore, the source can be classified as an SSS. Confidence contours for absorption column density and black-body temperature are shown in Fig. 1. The uncertainty ranges (90\% confidence) for the black-body parameters are relatively large, because the luminosity of the source had already

8 http://wWw.starlink.rl.ac.uk/gaia 


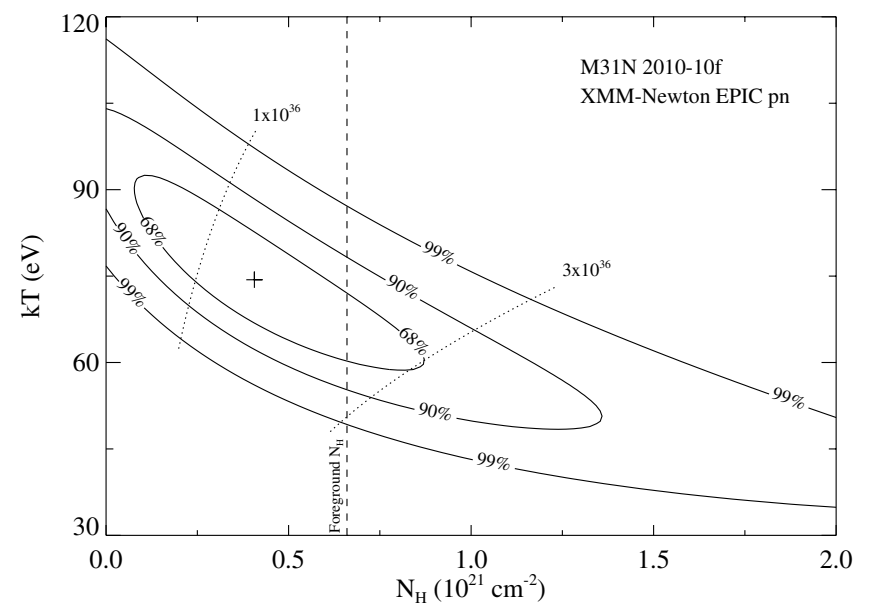

Fig. 1. Column density $\left(N_{\mathrm{H}}\right)$ - temperature $(k T)$ contours inferred from the simultaneous black body fit to the XMM-Newton EPIC pn spectra of M31N 2010-10f. Indicated are the formal best-fit parameters (cross), the lines of constant X-ray luminosity (0.2-10.0 keV, dotted lines), and the Galactic foreground absorption (dashed line).

declined significantly by the time of the XMM-Newton observations (see Table 1). Unfortunately, the Chandra HRC-I detector has no energy resolution to derive a spectrum from the initial, more luminous detections. Although the source was also bright during the Swift pointings, the shorter exposure time and smaller effective area of the XRT led to a combined spectrum in agreement with the XMM-Newton spectrum, but with larger uncertainties.

High-resolution spectra of Galactic novae (e.g. Nelson et al. 2008; Ness et al. 2011, 2012) clearly show a variety of absorption and emission features, underlining the fact that black body fits merely provide a qualitative parametrisation of SSS spectra and not a physically realistic model (see also Greiner et al. 1991; Kahabka \& van den Heuvel 1997). For individual nova SSS spectra, results based on assuming black body models have to be interpreted with great care, but general population trends appear to be describable by black-body temperatures (Henze et al. 2011).

Based on the black body model, we derived energy conversion factors for the different X-ray detectors in XSPEC, which were used to compute unabsorbed fluxes from the instrumental count rates listed in Table 1. The unabsorbed luminosities, which are given in Table 1, assume an M 31 distance of 780 kpc (Holland 1998; Stanek \& Garnavich 1998). No significant short-term variability was found in any of the individual observations.

As mentioned above, the discovery of the third SSS in an M 31 GC motivated a search for an optical counterpart, because one of the two other sources was identified with the first nova in the M 31 GC system (SQ2007, HPH2009). Archival optical data from Super-LOTIS showed Bol 126 at a constant $R=$ $16.7 \pm 0.1 \mathrm{mag}$ during the time from 2007 until the beginning of October 2010 and from November 2010 onward. During October 2010, the brightness of the GC experienced a significant increase by about one magnitude (see Table 2). Figure 2 shows that observations from five different telescopes indicate a potential nova outburst. This nova candidate is hereafter called M31N 2010-10f, following the naming convention described in Pietsch et al. (2007).

In Fig. 3 we illustrate the good agreement between the position of M31N 2010-10f, as inferred from the PS1 difference image, and the GC Bol 126. Light curves (in $r_{\mathrm{p} 1}$ and $i_{\mathrm{p} 1}$ AB-magnitudes) of M31N 2010-10f from PAndromeda data are shown in Fig. 4.

Assuming the quiescence magnitude of Bol 126 as given above, we computed the magnitudes of M31N 2010-10f using the standard formula. The results are given in Table 2 for the duration of the outburst and illustrated in Fig. 5 together with the $\mathrm{X}$-ray light curve. Using our high-cadence observational coverage, we could determine the time of the nova outburst with high precision to MJD $=(55480.51 \pm 0.05) \mathrm{d}$. This assumes that the first Xingming observation in Fig. 5 detected the beginning of the outburst, whereas the earlier PAndromeda observation (see Fig. 4) saw Bol 126 still at quiescence. The observed peak magnitudes of M31N 2010-10f are $R=(16.1 \pm 0.3)$ mag, detected in a Super-LOTIS observation on MJD $=55481.47$ (see Table 2), as well as $r=(15.512 \pm 0.009) \mathrm{mag}$ and $i=(15.602 \pm 0.009)$ mag from slightly earlier PAndromeda observations on MJD = 55481.34 and 55481.35 (see Table 3), respectively.

The PAndromeda light curve provides the data points closest to the outburst maximum of the nova and therefore these data are most suited to determine $t_{2}$, which is the time (in days) for the nova to decline by 2 magnitudes:

$m_{\mathrm{t} 2}=-2.5 \log \left(\left(F_{\mathrm{GC}}+10^{-0.4 \times 2 \operatorname{mag}}\left(F_{\max }-F_{\mathrm{GC}}\right)\right) / 3631 \mathrm{Jy}\right)$.

Using those points in the light curve that have the most similar brightness to $m_{t 2}$ gave upper limits of $t_{2, R} \leq 3.12 \mathrm{~d}$ and $t_{2, I} \leq$ $2.12 \mathrm{~d}$, making M31N 2010-10f a very fast nova in the classification system of Payne-Gaposchkin (1964). The brightness of the cluster was determined by aperture photometry to be $F_{\mathrm{R}, \mathrm{GC}}=$ $63.805 \times 10^{-5} \mathrm{Jy}$ and $F_{\mathrm{I}, \mathrm{GC}}=77.462 \times 10^{-5} \mathrm{Jy}(16.89 \mathrm{mag}$ and $16.68 \mathrm{mag}$ ).

With respect to the derived outburst date, the X-ray time scales of the nova can be estimated as follows: SSS turn-on time $t_{\text {on }}=(19 \pm 3) \mathrm{d}$ and turn-off time $t_{\text {off }}=(40 \pm 1) \mathrm{d}$. While $t_{\text {on }}$ is clearly constrained (see Table 1 ), $t_{\mathrm{Off}}$ is not as straightforward to determine. Between the second-last Swift observation and the second Chandra observation the luminosity of the SSS dropped by an order of magnitude (see Table 1 and Fig. 5). We interpret this observation as the end of the constant bolometric luminosity phase (MacDonald et al. 1985; Sala \& Hernanz 2005), which indicates the cessation of stable hydrogen burning (Hachisu \& Kato 2010).

Following our initial discovery alert (Henze et al. 2010a), Shafter et al. (2010) carried out optical spectroscopy to confirm the nova candidate. However, in their observation about $32 \mathrm{~d}$ after outburst they found no obvious Balmer emission lines that would indicate a recent nova. Such a short visibility in particular in $\mathrm{H} \alpha$ is unusual for an M 31 nova and might appear troubling at first. However, in the following we will outline how this fits into the picture that has emerged for M31N 2010-10f.

M31N 2010-10f was a very rapidly evolving object, much more so than the first M 31 GC nova (M31N 2007-06b in Bol 111), which indeed was found to show strong $\mathrm{H} \alpha$ emission for at least five weeks post-discovery (SQ2007). But even compared to the relatively fast M31N 2007-06b, the X-ray time scales for M31N 2010-10f were significantly shorter: SSS turnon time $(19 \pm 3) \mathrm{d}$ vs. $(87 \pm 54) \mathrm{d}$ and turn-off time $(40 \pm 1) \mathrm{d}$ vs. $(452 \pm 57) \mathrm{d}$. There is only an upper limit of $t_{2, R}<18 \mathrm{~d}$ known for M31N 2007-06b to compare to the very fast $t_{2, R} \leq 3.12 \mathrm{~d}$ of M31N 2010-10f, but in Henze et al. (2011) we found that short turn-on times for M 31 novae were correlated with fast expansion velocities of the ejected envelope as well as with rapid optical declines. Ciardullo et al. (1990a) noted that after the maximum $\mathrm{H} \alpha$ light of a nova (which occurs after the optical peak) 


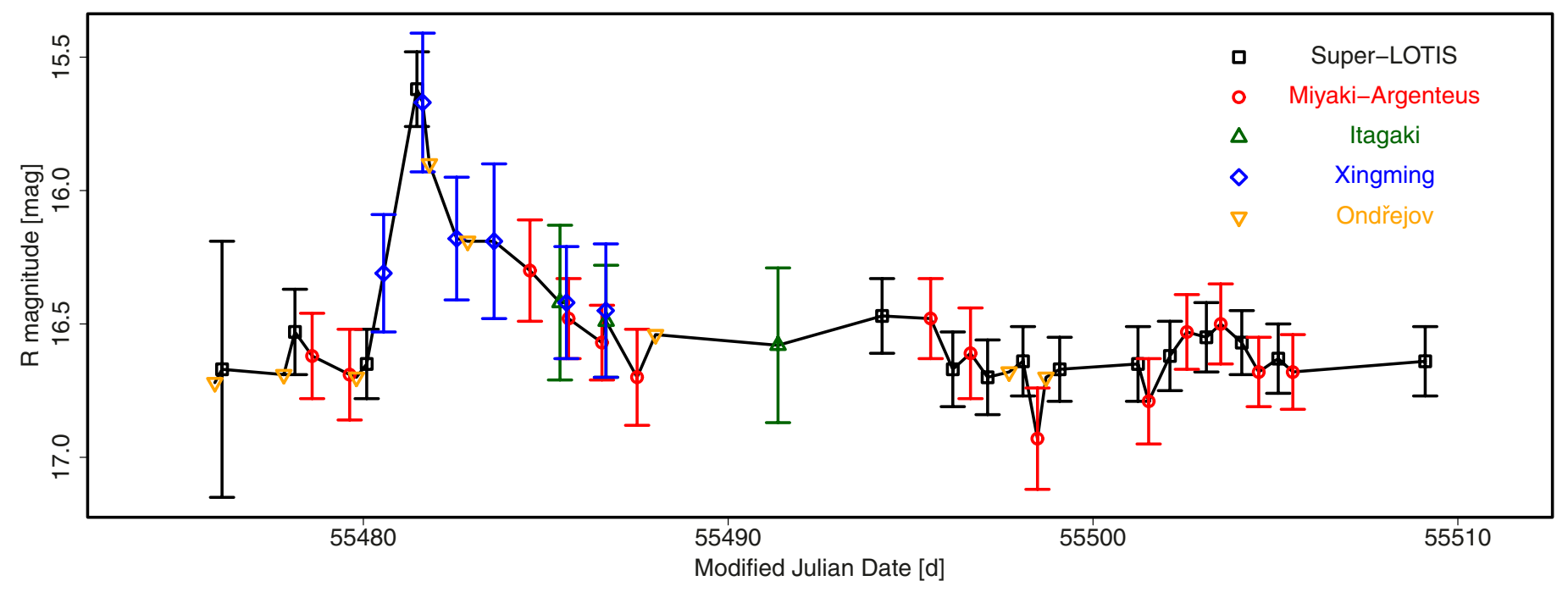

Fig. 2. Optical $R$ band light curve of Bol 126 consisting of data from five observatories indicated by different colours and symbols. One sigma confidence ranges are indicated by error bars. For the Ondřejov data the error ranges are about the size of the symbols.

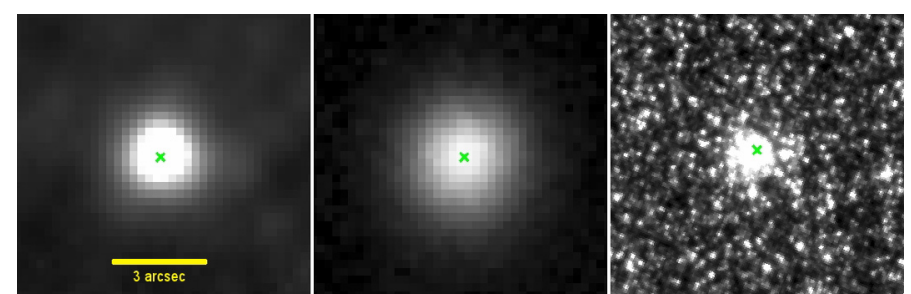

Fig. 3. Astrometric agreement of M31N 2010-10f (green cross) and the GC Bol 126 in a PS1 image (left) and a HST image (right) from ACS $814 \mathrm{~nm}$ archive data of HST Cycle 18 proposal 12058 by Dalcanton, J. The middle image shows the PS1 difference frame in $r_{\mathrm{P} 1}$ at the peak of the nova. The GC was subtracted in this frame and the image was used to compute the position of the nova as indicated by the green crosses in this figure. All three images have the same size $\left(\right.$ FOV $\left.9^{\prime \prime} \times 9^{\prime \prime}\right)$.

the decline in $\mathrm{H} \alpha$ matched the decline in broad band $B$ magnitude. Because our $R$ band includes the $\mathrm{H} \alpha$ line, we can assume that M31N 2010-10f would have experienced an $t_{2, \mathrm{H} \alpha} \lesssim 3 \mathrm{~d}$. Although Shafter et al. (2010) give no detection threshold for their observations, we assume that even from a bright $\mathrm{H} \alpha$ peak magnitude M31N 2010-10f could have faded sufficiently fast to be not detectable anymore in their measurements.

Moreover, according to current understanding (e.g. Hachisu $\&$ Kato 2006) the delay of the SSS turn-on with respect to the optical maximum provides an indirect hint for the presence of an expanding envelope (which earlier $\mathrm{H} \alpha$ observations should have detected) that at a certain time becomes optically thin to soft X-rays. Therefore, even without a confirmation and classification based on $\mathrm{H} \alpha$ detections or optical spectra, all evidence strongly indicates a nova outburst. The signature of the optical transient fits the amplitude as well as the shape of a nova outburst and the X-ray spectrum points towards a nuclear burning WD. There is no other type of object known that has these observable properties. Consequenly, we interpret our observations as revealing the outburst of a nova in the GC Bol 126.

Although there is an irregular variable present in the Wendelstein Calar Alto Pixellensing Project (WeCAPP) catalogue of Fliri et al. (2006) at a position only 0.35 away from Bol 126, a quick inspection of the corresponding light curve did not reveal a previous nova outburst. Similarly, no signatures of

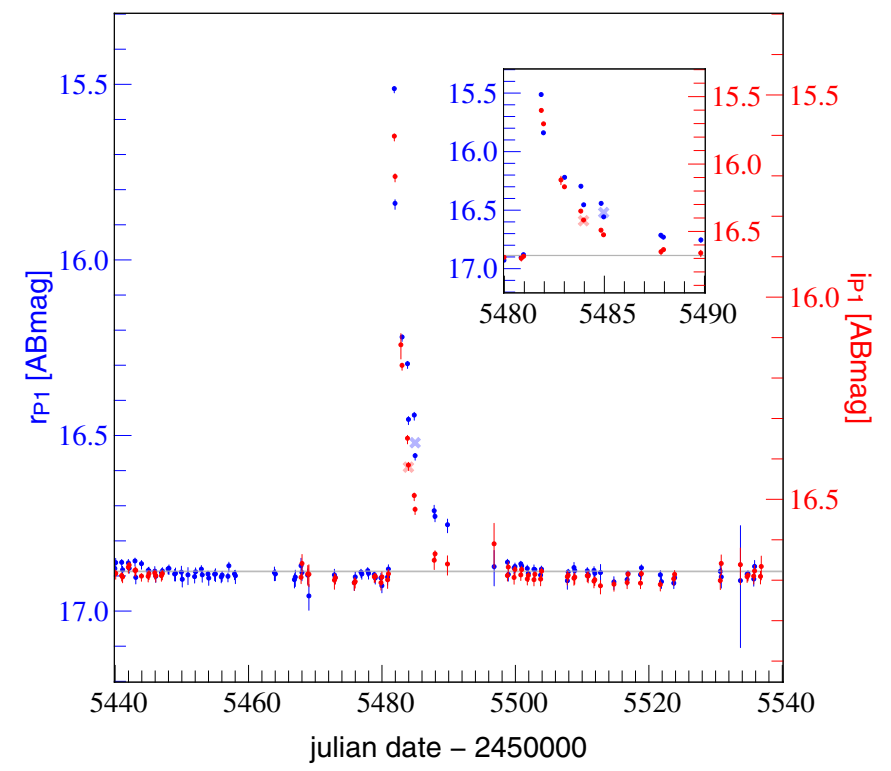

Fig. 4. Light curve of nova $M 31 N$ 2010-10f as measured from the PAndromeda data. The $r_{\mathrm{P} 1}$ and $i_{\mathrm{P} 1}$-band $\mathrm{AB}$-magnitudes and their errors are shown as blue and red data points and error bars. The measurements include the brightness of the globular cluster $\left(r_{\mathrm{P} 1}=16.89\right.$ and $i_{\mathrm{P} 1}=16.68 \mathrm{mag}$ ). The two large crosses in light blue and red show the estimated brightness and point in time $t_{2}$ where the nova faded by 2 magnitudes.

additional $\mathrm{CNe}$ were found in the WeCAPP light curves of GCs from the catalogues of Galleti et al. (2004) and Peacock et al. (2010) (see also Sect. 4.2).

An additional optical light curve of Bol 126 is given by Cao et al. (2012) based on Palomar Transient Factory data. These authors confirm the outburst and their light curve plots agree with our classification of M31N 2010-10f as a very fast nova.

\section{Discussion}

\subsection{Properties of nova M31N 2010-10 and M 31 GC novae}

M31N 2010-10f is only the second confirmed nova in the M 31 GC system to date. Its observational properties in the 


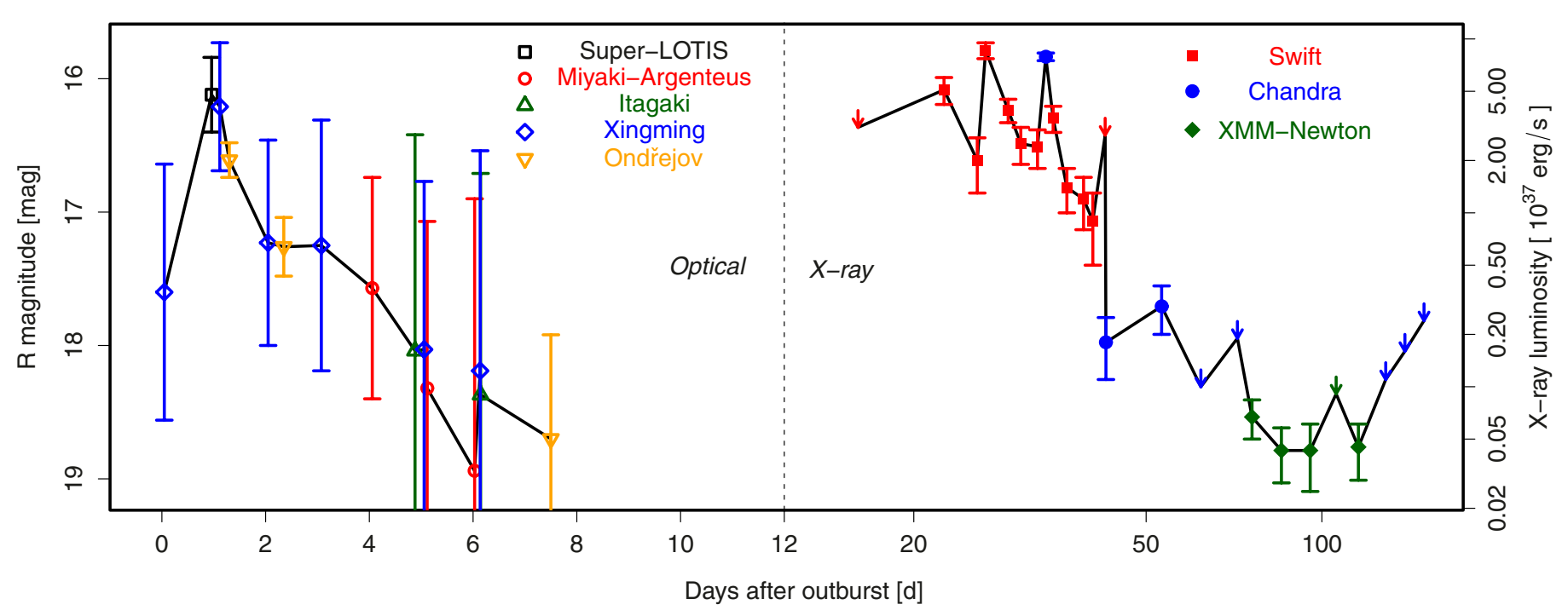

Fig. 5. Light curve of nova M31N 2010-10f in the optical $R$ band (left part, nova magnitude assuming Bol 126 having constant $R=16.7$ mag) and in the X-ray range (right part), assuming a nova outburst on MJD $=55480.32$ (see text). Note the logarithmic luminosity scale for the X-ray light curve and that the scale of the ordinate changes from linear to logarithmic at day 12. Error bars show $1 \sigma$ confidence range, down-pointing arrows indicate $3 \sigma$ upper limits. Data from different observatories are colour- and symbol-coded.

optical (relatively bright maximum magnitude, fast decline) and X-rays (short SSS duration, high black-body temperature) coherently point towards an underlying massive WD (see e.g. Livio 1992; Sala \& Hernanz 2005; Hachisu \& Kato 2006, 2010). By comparing the models of Hachisu \& Kato (2006), we estimated $M_{\mathrm{WD}} \gtrsim 1.3 M_{\odot}$. The relations between the measured properties $k T, t_{\mathrm{on}}, t_{\mathrm{off}}$ and $t_{2, R}$ do not deviate significantly from the general population trends presented in Henze et al. (2011).

Since binary systems with massive WDs are expected to be found in stellar populations younger than those of GCs (e.g. Della Valle \& Livio 1998; Henze et al. 2011) the properties of M31N 2010-10f are a noteworthy finding. It becomes even more remarkable, because the first M 31 GC nova, M31N 2007-06b in Bol 111, displayed features normally associated with young stellar populations $(\mathrm{He} / \mathrm{N}$ spectrum and broad Balmer emission lines, see SQ2007) and the X-ray properties of a third, putative GC nova (in Bol 194) reported in HPH2009 also indicate a massive WD (short SSS duration, high black-body temperature). All three GCs are old systems (age $>3 \mathrm{Gyr}$ ) with low metallicities of $[\mathrm{Fe} / \mathrm{H}]<-1.0$ (see e.g. Strader et al. 2011, and references therein).

The trend within GC novae towards hot, short SSS stages is unlikely to be caused by observational selection effects. In the field, novae with massive WDs are dominating the observed mass distribution because of their short recurrence times (e.g. Truran \& Livio 1986). However, those novae have also much shorter SSS durations and require high-cadence monitorings like our programme to find them. On the other hand, novae with low-mass WDs are visible in X-rays for years, some even for a decade (see Henze et al. 2011). Those objects would have been detected in the combined X-ray data from extensive monitoring of the M 31 central area (Henze et al. 2010b, 2011). In the optical, slow novae are considerably more difficult to detect against the GC background, as they are optically fainter.

The non-detection of slow novae in M 31 GCs together with the similarities between the known novae might therefore present a challenge to the current understanding of nova populations. This underlines the importance and wide discovery space of a regular monitoring of our neighbour galaxy with $\mathrm{X}$-ray telescopes, in particular if undertaken with high-cadence observations.

A possible answer to why only fast novae have been discovered in M 31 GCs so far might be found in a recent suggestion by Maccarone \& Zurek (2012) on how to enhance helium abundances in GCs. They discuss the impact on GC abundances of He-rich ejecta from novae powered by accretion of the intracluster medium (ICM) onto massive WDs (see also Naiman et al. 2011). The scenario of nova outbursts powered by accretion of interstellar matter was already mentioned by Della Valle et al. (2005) while examining type Ia supernovae (SNe Ia) rates in radio galaxies. According to the estimate of Maccarone \& Zurek (2012), there should be a large number of nova outbursts in GCs due to ICM accretion, but many of them will probably be obscured by the same high-density ICM from which they are accreting. Interestingly, this scenario should favour (massive) ONe WDs, as such systems form first, and could therefore explain the observations.

\subsection{The nova rate in M 31 GCs from the optical WeCAPP survey}

To determine a nova rate in M 31 GCs from the WeCAPP optical survey data, we used as reference the catalogue of Peacock et al. (2010). From a total of 572 confirmed GCs in the catalogue (416 old and 156 young GCs), 80 overlap with the WeCAPP field $\left(\mathrm{RA}=00^{\mathrm{h}} 41^{\mathrm{m}} 58.1^{\mathrm{s}}\right.$ to $00^{\mathrm{h}} 43^{\mathrm{m}} 29.4^{\mathrm{s}}$, Dec $=+41^{\circ} 07^{\prime} 43^{\prime \prime}$ to $+41^{\circ} 24^{\prime} 23^{\prime \prime}$; J2000.). For 78 GCs we were able to derive light curves in the $R$ band in our WeCAPP data, whereas two objects are too close to a saturated star to obtain reliable measurements. The coordinates given in Peacock et al. (2010) agree within an accuracy of less than $0 . .5$ with the centroids on the source positions of the WeCAPP frames (see Lee et al. 2012b).

We carried out a search for additional nova outbursts, according to the criteria described in Lee et al. (2012b), on the 78 remaining GC positions. We applied a slightly modified asymmetry criterion using $10 \sigma$ outliers and $a>6$ (an empirical 


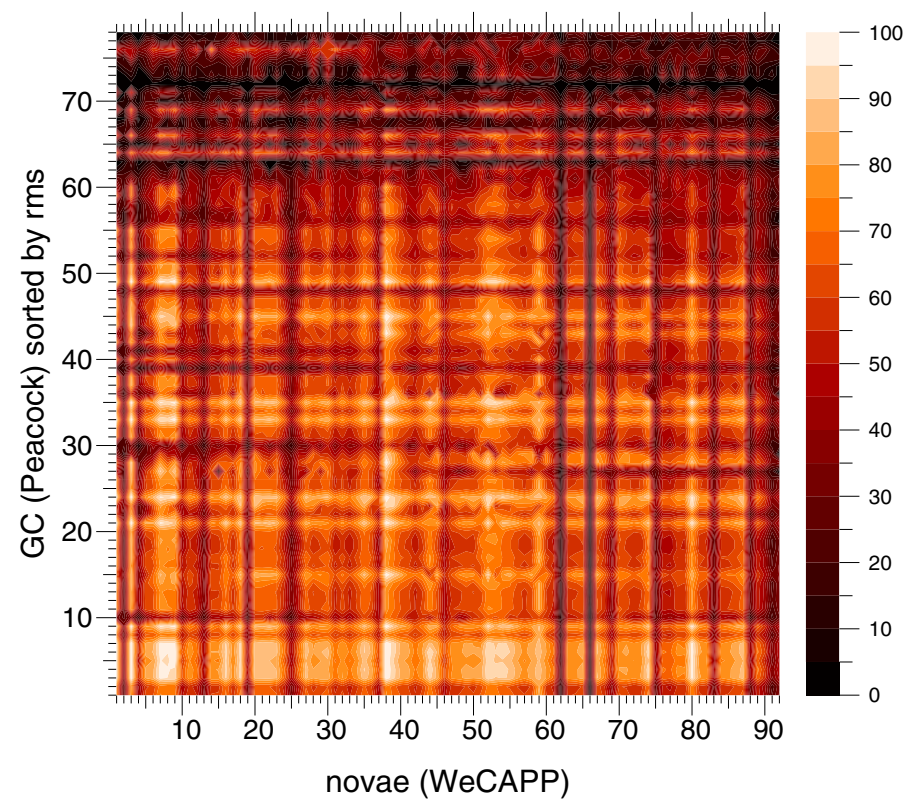

Fig. 6. Detection efficiency in percent (see scale) depending on 78 individual GC and 92 different novae types. The GCs, from the catalogue of Peacock et al. (2010), are sorted by increasing rms of their light curves. The light curve of M31N 2010-10f is number 92.

asymmetry parameter describing the balance between outliers in both directions) only for light curves that had at least seven upper outliers. With these criteria, no new nova was detected in the WeCAPP light curves of the $78 \mathrm{GCs}$, whereas all 91 WeCAPP novae and M31N 2010-10f were found.

To determine a detection efficiency we performed Monte Carlo simulations using our 91 WeCAPP novae and the M31N 2010-10f light curve as a sample and interpolating linearly between the magnitude values of the data points. For all 78 individual GCs each of the 92 nova light curves was simulated $10^{4}$ times, where we equally distributed the maximum time of the different WeCAPP novae over the WeCAPP survey time between Julian dates of 2450685.5 and 2454535.3.

The results of the simulation are illustrated in Fig. 6, which shows that the detection efficiency mainly depends on the individual nova light curve (vertical stripes) and the rms of the GC light curves (horizontal stripes). Nova light curves can differ strongly in maximum brightness and duration, i.e. the detection window varies. For the GC light curves Fig. 6 shows that the detection efficiency tends to decrease with increasing rms. Deviations from this trend in individual GCs are caused by the quite diverse time sampling and different noise quality of their light curves. In particular, some GCs show an intrinsic variability that reduces nova detections with our criteria. Therefore the measurable nova rate can differ, depending on the type of novae present in the particular GC, and Monte Carlo simulations were necessary to estimate the detection efficiency.

For nova light curves similar to that of M31N 2010-10f the mean detection efficiency is $27 \%$. With only one detected nova in $78 \mathrm{GCs}$ during the WeCAPP survey period of $10.54 \mathrm{yr}$, this results in a tentative nova rate of 0.0045 novae $\mathrm{yr}^{-1} \mathrm{GC}^{-1}$. The corresponding $95 \%$ confidence Poisson upper limit is 0.025 novae $\mathrm{yr}^{-1} \mathrm{GC}^{-1}$. Surveying all confirmed GCs of Peacock et al. (2010) for one year, we would expect two to three novae to be detected. For all nova light curves and all confirmed GCs, the mean detection efficiency becomes $51 \%$, which reduces the above rate estimate and confidence limit by a factor of about two.
For the final data release of Pan-STARRS 1 with its key project PAndromeda we expect to further constrain these numbers.

\subsection{The nova rate in M 31 GCs from the X-ray monitoring}

Assuming that both SSSs presented in HPH2009 were actually novae, we here estimate the nova rate for the M $31 \mathrm{GC}$ system based on X-ray observations. In five recent X-ray monitoring campaigns aimed at the M 31 central region we detected three $\mathrm{GC}$ novae. The campaigns considered here were carried out with a $10 \mathrm{~d}$ cadence and are summarised in Henze et al. $(2011 ; 2007 / 8$ and 2008/9) and Henze et al. (in prep.; 2009/10, 2010/11, and 2011/12). The first dedicated monitoring campaign for X-ray emission from M 31 novae (reported in Henze et al. 2010b) had a lower cadence of about $30 \mathrm{~d}$ and is therefore not included in our estimate. Since the two sources reported in HPH2009 were only found at large off-axis angles in the Chandra HRC-I observations of the monitoring (the XMM-Newton EPIC field of view is slightly smaller), only HRC-I pointings are considered here. The Chandra part of each individual campaign covered a time span of one to two months, resulting in a total effective survey time of nine months.

Recently, Henze et al. (2011) compiled a catalogue of 60 novae with an X-ray counterpart in M 31. Even the fastest of these objects generally had an SSS duration of $\sim 20-30 \mathrm{~d}$. Therefore, we assume that a $10 \mathrm{~d}$ cadence monitoring should be able to detect all SSS counterparts of hypothetical GC novae during the time of the coverage. This means that in contrast to our estimate in HPH2009, the SSS duration of the nova is not a critical parameter.

Of the approximately 650 confirmed GCs known in M 31 today (see the RBC; Galleti et al. 2004), 160 are located within the field of view of the Chandra HRC-I (assuming a 16' radius around the M 31 centre). Of those, we expect about $50 \%$ to be located within or behind the M 31 disk, as current GC catalogues appear to be complete except for a few objects located behind dust lanes (Caldwell et al. 2011). This assumption is supported by a comparison of GC reddening estimates from the work of Fan et al. (2008) with an M 31 reddening map derived by Montalto et al. (2009), for which we found approximately half of the GCs to have higher extinctions than the M 31 disk around their position (see also Trudolyubov \& Priedhorsky 2004).

Our nova rate estimate needs to take into account the higher extinction for GCs in and particularly behind the M 31 disk, as supersoft X-rays are strongly attenuated by a high foreground column density. Additional absorption takes place within the intra-cluster medium and in the matter ejected by the current or previous nova outbursts. These latter effects are difficult to quantify. Here, we assume that the combined extinction for GC novae reduces the sample of GCs in which an SSS could be found in our monitoring by $\sim 50 \%$. Therefore, the detection of three novae in about 80 GCs within nine months leads to a rate of $\sim 0.05$ novae $\mathrm{yr}^{-1} \mathrm{GC}^{-1}$.

Another effect that might influence whether the SSS state of a potential nova is detected or not, is the presence of other $\mathrm{X}$-ray sources in the GC. About 40 confirmed GCs from the RBC are detected in X-rays within the Chandra HRC field of view. This number is slightly reduced for the smaller XMM-Newton EPIC field. We constructed long-term X-ray light curves for XMM-Newton and Chandra for each GC. In none of these light curves we did find unambiguous evidence for an additional outburst component. In case of XMM-Newton, using the spectral 
resolution of the EPIC detectors, we furthermore studied the evolution of hardness ratios (X-ray colours) over time, searching for the signature of a supersoft, transient source. No such event could be identified.

However, of the $40 \mathrm{HRC}$ sources, four have average luminosities exceeding $10^{38} \mathrm{erg} \mathrm{s}^{-1}$ and show intrinsic variability (they are mostly black hole binary candidates, see e.g. Barnard et al. 2012). These properties would make it difficult to detect the presence of a fainter nova with a luminosity of only a few $10^{37} \mathrm{erg} \mathrm{s}^{-1}$. Although the hardness ratios of these sources are relatively stable over time, and no hint of a supersoft excess could be seen, we cannot exclude that a nova outburst could have been hidden in the existing variability. In the present context, the small number of four sources does not change the above estimate of about $80 \mathrm{GCs}$, in which novae could have been found, and therefore has no impact on our detection rate estimate of $\sim 0.05$ novae $\mathrm{yr}^{-1} \mathrm{GC}^{-1}$.

Taken at face value, the nova rate derived from our X-ray monitoring exceeds the optical estimate based on the WeCAPP data (see Sect.4.2) by about one order of magnitude. This demonstrates the advantages of X-ray surveys over optical observations when searching for novae in GCs. However, that nova M31N 2007-06b (SQ2007) probably was only missed by the WeCAPP project because it was outside the field of view. The $\mathrm{X}$-ray rate is also higher by a factor of 10 or 25 compared to upper limits from earlier optical surveys (e.g. $0.005 \mathrm{yr}^{-1} \mathrm{GC}^{-1}$ in Tomaney et al. 1992) or simple estimates based on nova rates in elliptical galaxies (0.002, see HPH2009 and references therein), respectively.

Applying Poisson statistics, our X-ray discoveries (lower 95\% confidence limit: 0.01 novae $\mathrm{yr}^{-1} \mathrm{GC}^{-1}$ ) are still consistent within the $95 \%$ confidence limits with the WeCAPP estimate. However, this does not agree with the other two estimates within the $95 \%$ confidence range (which corresponds to $0.8-11.7$ novae $\mathrm{yr}^{-1}$ for the entire GC system).

Even without taking into account any extinction effects, our results remain significant. Considering all 160 GCs in our field of view still produces a (factor two lower) nova rate that is higher by a factor of 5 or 12 compared to the optical upper limits for M 31 GCs and elliptical galaxy rates and excludes those estimates on the $95 \%$ confidence level.

For a conservative estimate, we assume that by chance the entire M 31 GC nova production took place in only 80/650 $12 \%$ of GCs, during the time of our monitoring. This approach leads to a rate of 0.006 novae $\mathrm{yr}^{-1} \mathrm{GC}^{-1}$ and a $95 \%$ confidence lower limit of 0.001 novae $\mathrm{yr}^{-1} \mathrm{GC}^{-1}$. These numbers are comparable to the upper limits from optical surveys and the rates in ellipticals. However, the probability for this scenario is only $0.2 \%$.

\subsection{Expected nova rates in M 31 GCs and the overabundance of binary systems}

Now we estimate the expected nova rate for the M 31 GC system assuming that in GCs $\mathrm{CNe}$ are produced with the same efficiency as in the field. For this, we compared the $K$ band magnitudes of M 31 and its GC system and used this relation to scale the overall M 31 nova rate. The RBC includes infrared measurements (from the 2MASS project; Skrutskie et al. 2006) for about 350 confirmed GCs with a total magnitude of $K \sim 7.1 \mathrm{mag}$. We assume that the remaining about 300 confirmed GCs were too faint to be detected by 2 MASS (detection limit $K \sim 15.3$ mag; Cutri et al. 2003), because they show (a) a similar spatial distribution as the GCs with $K$ band magnitude and (b) a significantly fainter average magnitude in the $R$ and $I$ bands. Adding 300 objects with magnitudes of $K=16-17$ mag only changes the total magnitude to $K \sim 7.0 \mathrm{mag}$, which we used as a conservative estimate for all confirmed M 31 GCs. For an M 31 total $K$ band magnitude of 0.98 (2MASS; Skrutskie et al. 2006), this means that M 31 is about a factor of 250 brighter (in the $K$ band) than its GC system.

We scaled the total M 31 nova rate of about $65 \mathrm{yr}^{-1}$ (Darnley et al. 2006) with the luminosity to $65 / 250=0.26$ novae $\mathrm{yr}^{-1}$ for the whole GC system. Assuming that the M 31 nova rate is dominated by an old (bulge) stellar population (e.g. Ciardullo et al. 1987; Capaccioli et al. 1989), the extrapolation from a total rate to a rate in the old populations of GCs is justified. Under the assumptions of Sect. 4.3 the observed rate is three novae in nine months of effective survey time or four novae $\mathrm{yr}^{-1}$. Therefore, the derived GC nova rate might be higher than measured for the host galaxy by a factor of $4 / 0.26 \sim 15$, with the $95 \%$ confidence lower limit allowing for an enhancement by at least a factor of $0.8 / 0.26 \sim 3$.

The above estimates indicate that an additional binaryforming mechanism might exist in GCs to increase the nova rate. One alternative scenario, increased accretion onto compact objects in GCs, is discussed in Sect.4.1. It is long known that low-mass X-ray binaries (LMXBs) are significantly overabundant in M 31 GCs (e.g. Fan et al. 2005), which was explained by tidal captures of main-sequence stars by neutron stars (Clark 1975; Fabian et al. 1975). These processes are also expected to increase the number of WD binaries (Hut \& Verbunt 1983). Indeed, Galactic GCs have been found to harbour a large number of cataclysmic variables (CVs), the majority of which are strongly suspected to have been formed dynamically (e.g. Maccarone \& Knigge 2007; Knigge 2011).

Despite the recent progress in finding novae in GCs, made possible by the regular X-ray monitoring of $\mathrm{M} 31$, the number of these objects is still small. Finding them is important for various reasons: (i) knowing the nova rate in GCs allows for a more accurate estimate of the total nova rate in a galaxy; (ii) comparing sample properties for field- and GC novae might provide additional information on $\mathrm{CV}$ formation processes; (iii) novae could play an important role in the ecosystem of the GC, such as removing part of the intra-cluster medium (Moore \& Bildsten 2011), enhancing its helium abundance (Maccarone \& Zurek 2012) or creating unusual emission spectra (Ripamonti \& Mapelli 2012); (iv) as potential progenitors of SNe Ia novae could help to access the SN Ia rate in GCs (e.g. Voss \& Nelemans 2012).

Finally, a recent result by Peacock et al. (2010) is worth to be underlined. These authors reported a possible indication for a nova outburst in the M 31 GC Bol 383 in August 1991. At this time, observations by Reed et al. (1992) showed Bol 383 about 0.5 mag brighter than measured before and afterwards. Peacock et al. (2010) concluded that a transient with $M_{V} \sim 8$ and a bluer colour index than the GC would have been needed to explain this phenomenon. No X-ray source is known in this GC. This cluster is also an old one (Strader et al. 2011). We encourage optical observers to check their archives, not only for additional observations of Bol 383 during the time of August 1991, but for possible indications of nova outbursts in GCs in general.

\section{Summary}

We presented the discovery and properties of the second confirmed $\mathrm{CN}$ in a $\mathrm{M} 31 \mathrm{GC}$ based on high-cadence optical and X-ray monitoring observations. This object, 
named M31N 2010-10f, exhibits the characteristics of harbouring a massive WD $\left(M_{\mathrm{WD}} \gtrsim 1.3 M_{\odot}\right)$ in its X-ray (high blackbody temperature, short SSS phase) as well as in the optical properties (relatively bright peak magnitude, fast decline). Together with two additional GC novae (one suspected) from earlier work (HPH2009), M31N 2010-10f allowed us to estimate the M 31 GC nova rate based on our regular X-ray monitoring. We found a tentative rate of 0.05 novae $\mathrm{yr}^{-1} \mathrm{GC}^{-1}$ that is about an order of magnitude higher than expected from stellar evolution or upper limits from earlier optical surveys. Complementing analyses of the recent WeCAPP optical survey provided a lower nova rate (by about an order of magnitude), but did not contain one of the two known optical GC novae in its field of view and is still consistent with the X-ray estimate on the $95 \%$ level. Furthermore, we estimated that the observed luminosity-specific nova rate is at least (on the $95 \%$ confidence level) a factor of three higher in M 31 GCs than for the (similarly old) bulge population. These results further underline the need for additional processes in GCs, leading to dynamical binary formation or more effective accretion onto compact objects.

Unlike M31N 2010-10f, most novae with an SSS counterpart in $\mathrm{M} 31$ were discovered in the optical before their X-ray emission was detected (see e.g. Pietsch et al. 2005a, 2007; Henze et al. 2010b, 2011). This is due to quiescent M 31 novae being too faint to be observed with small optical telescopes and nova surveys therefore search for the eponymous new stars. For novae in relatively bright GCs, though, the outburst signature is much more subtle (see Fig. 5) and can easily go unnoticed, as happened for M31N 2010-10f until its discovery as an SSS. X-ray monitoring surveys with high cadence provide a powerful method to discover $\mathrm{CNe}$ in GCs and study their individual properties and overall outburst rates.

Acknowledgements. The anonymous referee is acknowledged for constructive comments that helped to improve the clarity of the paper. We would like to thank K. Lutz for her help in setting up the optical data reduction pipeline. The $X M M-N e w t o n$ project is supported by the Bundesministerium für Wirtschaft und Technologie/Deutsches Zentrum für Luft- und Raumfahrt (BMWI/DLR FKZ 50 OX 0001) and the Max Planck Society. We would like to thank the Swift team for the scheduling of the ToO observations. M. Henze acknowledges support from the BMWI/DLR, FKZ 50 OR 1010. The work of K. Hornoch was supported by the project RVO:67985815. We thank M. Wolf, P. Hornochová, P. Kušnirák, and P. Zasche for their assistance with acquiring of the observations at the Ondřejov Observatory. The PS1 Surveys have been made possible through contributions of the Institute for Astronomy at the University of Hawaii in Manoa, the Pan-STARRS Project Office, the Max Planck Society and its participating institutes, the Max Planck Institute for Astronomy, Heidelberg and the Max Planck Institute for Extraterrestrial Physics, Garching, the Johns Hopkins University, the University of Durham, the University of Edinburgh, the Queens University of Belfast, the Harvard-Smithsonian Center for Astrophysics, and the Los Cumbres Observatory Global Telescope Network, Incorporated, and the National Central University of Taiwan.

\section{References}

Alard, C., \& Lupton, R. H. 1998, ApJ, 503, 325

Arnaud, K. A. 1996, in Astronomical Data Analysis Software and Systems V, eds. G. H. Jacoby, \& J. Barnes, ASP Conf. Ser., 101, 17

Barnard, R., Garcia, M., \& Murray, S. S. 2012, ApJ, 757, 40

Bertin, E. 2006, in Astronomical Data Analysis Software and Systems XV, eds. C. Gabriel, C. Arviset, D. Ponz, \& S. Enrique, ASP Conf. Ser., 351, 112

Bertin, E., \& Arnouts, S. 1996, A\&AS, 117, 393

Bertin, E., Mellier, Y., Radovich, M., et al. 2002, in Astronomical Data Analysis Software and Systems XI, eds. D. A. Bohlender, D. Durand, \& T. H. Handley, ASP Conf. Ser., 281, 228

Blackburn, J. K. 1995, in Astronomical Data Analysis Software and Systems IV, eds. R. A. Shaw, H. E. Payne, \& J. J. E. Hayes, ASP Conf. Ser., 77, 367
Bode, M. F., \& Evans, A. 2008, Classical Novae, eds. M. F. Bode, \& A. Evans Burrows, D. N., Hill, J. E., Nousek, J. A., et al. 2005, Space Sci. Rev., 120, 165

Caldwell, N., Schiavon, R., Morrison, H., Rose, J. A., \& Harding, P. 2011, AJ, 141,61

Cao, Y., Kasliwal, M. M., Neill, J. D., et al. 2012, ApJ, 752, 133

Capaccioli, M., Della Valle, M., Rosino, L., \& D’Onofrio, M. 1989, AJ, 97, 1622

Ciardullo, R., Ford, H. C., Neill, J. D., Jacoby, G. H., \& Shafter, A. W. 1987, ApJ, 318, 520

Ciardullo, R., Shafter, A. W., Ford, H. C., et al. 1990a, ApJ, 356, 472

Ciardullo, R., Tamblyn, P., \& Phillips, A. C. 1990b, PASP, 102, 1113

Clark, G. W. 1975, ApJ, 199, L143

Cutri, R. M., Skrutskie, M. F., van Dyk, S., et al. 2003, 2MASS All Sky Catalog of point sources

Darnley, M. J., Bode, M. F., Kerins, E., et al. 2006, MNRAS, 369, 257

Della Valle, M., \& Livio, M. 1998, ApJ, 506, 818

Della Valle, M., Panagia, N., Padovani, P., et al. 2005, ApJ, 629, 750

Dieball, A., Long, K. S., Knigge, C., Thomson, G. S., \& Zurek, D. R. 2010, ApJ, 710,332

Dotani, T., Asai, K., \& Greiner, J. 1999, PASJ, 51, 519

Edmonds, P. D., Kahabka, P., \& Heinke, C. O. 2004, ApJ, 611, 413

Fabian, A. C., Pringle, J. E., \& Rees, M. J. 1975, MNRAS, 172, 15

Fan, Z., Ma, J., Zhou, X., et al. 2005, PASP, 117, 1236

Fan, Z., Ma, J., de Grijs, R., \& Zhou, X. 2008, MNRAS, 385, 1973

Fliri, J., Riffeser, A., Seitz, S., \& Bender, R. 2006, A\&A, 445, 423

Fruscione, A., McDowell, J. C., Allen, G. E., et al. 2006, in SPIE Conf. Ser., 6270

Gabriel, C., Denby, M., Fyfe, D. J., et al. 2004, in Astronomical Data Analysis Software and Systems (ADASS) XIII, eds. F. Ochsenbein, M. G. Allen, \& D. Egret, ASP Conf. Ser., 314, 759

Galleti, S., Federici, L., Bellazzini, M., Fusi Pecci, F., \& Macrina, S. 2004, A\&A, 416, 917

Gössl, C. A., \& Riffeser, A. 2002, A\&A, 381, 1095

Gössl, C. A., Drory, N., \& Snigula, J. 2004, in Astronomical Data Analysis Software and Systems (ADASS) XIII, eds. F. Ochsenbein, M. G. Allen, \& D. Egret, ASP Conf. Ser., 314, 456

Gregory, P. C., \& Loredo, T. J. 1992, ApJ, 398, 146

Greiner, J., Hasinger, G., \& Kahabka, P. 1991, A\&A, 246, L17

Hachisu, I., \& Kato, M. 2006, ApJS, 167, 59

Hachisu, I., \& Kato, M. 2010, ApJ, 709, 680

Henze, M., Meusinger, H., \& Pietsch, W. 2008, A\&A, 477, 67

Henze, M., Pietsch, W., Haberl, F., et al. 2009, A\&A, 500, 769 [HPH2009]

Henze, M., Pietsch, W., Burwitz, V., et al. 2010a, ATel, 3019, 1

Henze, M., Pietsch, W., Haberl, F., et al. 2010b, A\&A, 523, A89

Henze, M., Pietsch, W., Haberl, F., et al. 2011, A\&A, 533, A52

Holland, S. 1998, AJ, 115, 1916

Hut, P., \& Verbunt, F. 1983, Nature, 301, 587

Kaaret, P. 2002, ApJ, 578, 114

Kahabka, P., \& van den Heuvel, E. P. J. 1997, ARA\&A, 35, 69

Knigge, C. 2011 [arXiv: 1112.1074]

Kong, A. K. H., Garcia, M. R., Primini, F. A., et al. 2002, ApJ, 577, 738

Krautter, J. 2002, in Classical Nova Explosions, eds. M. Hernanz, \& J. José, Am. Inst. Phys. Conf. Ser., 637, 345

Lee, C.-H., Riffeser, A., Koppenhoefer, J., et al. 2012a, AJ, 143, 89

Lee, C.-H., Riffeser, A., Seitz, S., et al. 2012b, A\&A, 537, A43

Livio, M. 1992, ApJ, 393, 516

Luther. 1860, Astron. Nachr., 53, 293

Maccarone, T., \& Knigge, C. 2007, Astron. Geophys., 48, 050000

Maccarone, T. J., \& Zurek, D. R. 2012, MNRAS, 423, 2

MacDonald, J., Fujimoto, M. Y., \& Truran, J. W. 1985, ApJ, 294, 263

Massey, P., Olsen, K. A. G., Hodge, P. W., et al. 2006, AJ, 131, 2478

Montalto, M., Seitz, S., Riffeser, A., et al. 2009, A\&A, 507, 283

Moore, K., \& Bildsten, L. 2011, ApJ, 728, 81

Murray, S. S., Austin, G. K., Chappell, J. H., et al. 2000, in SPIE Conf. Ser. 4012, eds. J. E. Truemper, \& B. Aschenbach, 68

Naiman, J. P., Ramirez-Ruiz, E., \& Lin, D. N. C. 2011, ApJ, 735, 25

Nelson, T., Orio, M., Cassinelli, J. P., et al. 2008, ApJ, 673, 1067

Ness, J.-U., Osborne, J. P., Dobrotka, A., et al. 2011, ApJ, 733, 70

Ness, J.-U., Schaefer, B. E., Dobrotka, A., et al. 2012, ApJ, 745, 43

Payne-Gaposchkin, C. 1964, The galactic novae (New York: Dover Publication)

Peacock, M. B., Maccarone, T. J., Knigge, C., et al. 2010, MNRAS, 402, 803

Pietsch, W. 2010, Astron. Nachr., 331, 187

Pietsch, W., Fliri, J., Freyberg, M. J., et al. 2005a, A\&A, 442, 879

Pietsch, W., Freyberg, M., \& Haberl, F. 2005b, A\&A, 434, 483

Pietsch, W., Haberl, F., Sala, G., et al. 2007, A\&A, 465, 375

Pietsch, W., Henze, M., Burwitz, V., et al. 2010a, ATel, 3001, 1

Pietsch, W., Henze, M., Haberl, F., \& Burwitz, V. 2010b, ATel, 3038, 1 
A\&A 549, A120 (2013)

Pietsch, W., Henze, M., Haberl, F., \& Burwitz, V. 2010c, ATel, 3013, 1 Reed, L. G., Harris, G. L. H., \& Harris, W. E. 1992, AJ, 103, 824 Ripamonti, E., \& Mapelli, M. 2012, MNRAS, 423, 1144

Sala, G., \& Hernanz, M. 2005, A\&A, 439, 1061

Schwarz, G. J., Ness, J.-U., Osborne, J. P., et al. 2011, ApJS, 197, 31

Shafter, A. W., \& Irby, B. K. 2001, ApJ, 563, 749

Shafter, A. W., \& Quimby, R. M. 2007, ApJ, 671, L121 [SQ2007]

Shafter, A. W., Ciardullo, R., Darnley, M. J., Bode, M. F., \& Misselt, K. A. 2010, ATel, 3074, 1

Shara, M. M., Zurek, D. R., Baltz, E. A., Lauer, T. R., \& Silk, J. 2004, ApJ, 605, L117

Skrutskie, M. F., Cutri, R. M., Stiening, R., et al. 2006, AJ, 131, 1163

Stacey, W. S., Heinke, C. O., Elsner, R. F., et al. 2011, ApJ, 732, 46

Stanek, K. Z., \& Garnavich, P. M. 1998, ApJ, 503, L131

Starrfield, S. 1989, in Classical Novae, 39

Starrfield, S., Sparks, W. M., \& Truran, J. W. 1974, ApJS, 28, 247
Stiele, H., Pietsch, W., Haberl, F., et al. 2011, A\&A, 534, A55

Strader, J., Caldwell, N., \& Seth, A. C. 2011, AJ, 142, 8

Strüder, L., Briel, U., Dennerl, K., et al. 2001, A\&A, 365, L18

Tomaney, A., Crotts, A., \& Shafter, A. 1992, in BAAS, 24, 1237

Trudolyubov, S., \& Priedhorsky, W. 2004, ApJ, 616, 821

Trümper, J., Hasinger, G., Aschenbach, B., Braeuninger, H., \& Briel, U. G. 1991, Nature, 349, 579

Truran, J. W., \& Livio, M. 1986, ApJ, 308, 721

Tuchman, Y., \& Truran, J. W. 1998, ApJ, 503, 381

Turner, M. J. L., Abbey, A., Arnaud, M., et al. 2001, A\&A, 365, L27

Verbunt, F., Bunk, W., Hasinger, G., \& Johnston, H. M. 1995, A\&A, 300, 732

Voss, R., \& Nelemans, G. 2012, A\&A, 539, A77

Williams, B. F., Garcia, M. R., Kong, A. K. H., et al. 2004, ApJ, 609, 735

Williams, G. G., Milne, P. A., Park, H. S., et al. 2008, in AIP Conf. Ser., 1000, 535 
M. Henze et al.: X-rays reveal second M 31 globular cluster nova

Table 1. X-ray observations of nova M31N 2010-10f.

\begin{tabular}{|c|c|c|c|c|c|c|}
\hline Telescope/Instrument ${ }^{a}$ & ObsID & $\begin{array}{c}\text { Exp. time } \\
{[\mathrm{ks}]}\end{array}$ & $\begin{array}{l}\text { Date }^{c} \\
\text { [UT] }\end{array}$ & $\begin{array}{l}\Delta t^{d} \\
{[\mathrm{~d}]}\end{array}$ & $\begin{array}{c}\text { Count rate }^{e} \\
{\left[\mathrm{ct} \mathrm{s}^{-1}\right]}\end{array}$ & $\begin{array}{l}L_{0.2-10.0}{ }^{e} \\
{\left[\mathrm{erg} \mathrm{s}^{-1}\right]}\end{array}$ \\
\hline Swift XRT & 00031851001 & 2.6 & $2010-10-27.56$ & 16.05 & $<6.7 \times 10^{-3}$ & $<3.1 \times 10^{37}$ \\
\hline Swift XRT & 00031851002 & 4.8 & 2010-11-03.04 & 22.53 & $(1.1 \pm 0.2) \times 10^{-2}$ & $(5.1 \pm 0.9) \times 10^{37}$ \\
\hline Swift XRT & 00031861002 & 3.9 & 2010-11-06.20 & 25.69 & $(4.3 \pm 1.5) \times 10^{-3}$ & $(2.0 \pm 0.7) \times 10^{37}$ \\
\hline Swift XRT & 00031861001 & 7.7 & 2010-11-07.06 & 26.55 & $(1.9 \pm 0.2) \times 10^{-2}$ & $(8.6 \pm 0.9) \times 10^{37}$ \\
\hline Swift XRT & 00031861003 & 7.7 & 2010-11-09.53 & 29.02 & $(8.4 \pm 1.4) \times 10^{-3}$ & $(3.9 \pm 0.6) \times 10^{37}$ \\
\hline Swift XRT & 00031861004 & 7.9 & 2010-11-11.01 & 30.50 & $(5.5 \pm 1.2) \times 10^{-3}$ & $(2.5 \pm 0.6) \times 10^{37}$ \\
\hline Swift XRT & 00031861005 & 6.8 & 2010-11-13.09 & 32.58 & $(5.3 \pm 1.3) \times 10^{-3}$ & $(2.4 \pm 0.6) \times 10^{37}$ \\
\hline Chandra HRC-I & 12110 & 20.0 & 2010-11-14.17 & 33.66 & $(2.1 \pm 0.1) \times 10^{-2}$ & $(7.9 \pm 0.4) \times 10^{37}$ \\
\hline Swift XRT & 00031861006 & 6.8 & 2010-11-15.16 & 34.65 & $(7.7 \pm 1.3) \times 10^{-3}$ & $(3.5 \pm 0.6) \times 10^{37}$ \\
\hline Swift XRT & 00031861007 & 8.2 & $2010-11-17.09$ & 36.58 & $(3.0 \pm 0.9) \times 10^{-3}$ & $(1.4 \pm 0.4) \times 10^{37}$ \\
\hline Swift XRT & 00031861008 & 6.8 & 2010-11-19.58 & 39.07 & $(2.7 \pm 0.9) \times 10^{-3}$ & $(1.2 \pm 0.4) \times 10^{37}$ \\
\hline Swift XRT & 00031861009 & 8.2 & 2010-11-21.03 & 40.52 & $(1.9 \pm 0.9) \times 10^{-3}$ & $(0.9 \pm 0.4) \times 10^{37}$ \\
\hline Swift XRT & 00031861010 & 7.9 & 2010-11-23.05 & 42.54 & $<6.0 \times 10^{-3}$ & $<2.8 \times 10^{37}$ \\
\hline Chandra HRC-I & 12111 & 19.9 & 2010-11-23.18 & 42.67 & $(4.9 \pm 1.9) \times 10^{-4}$ & $(1.8 \pm 0.7) \times 10^{36}$ \\
\hline Chandra HRC-I & 12112 & 19.9 & 2010-12-03.66 & 53.15 & $(7.7 \pm 2.4) \times 10^{-4}$ & $(2.9 \pm 0.9) \times 10^{36}$ \\
\hline Chandra HRC-I & 12113 & 19.0 & 2010-12-12.56 & 62.05 & $<2.7 \times 10^{-4}$ & $<1.0 \times 10^{36}$ \\
\hline Chandra HRC-I & 12114 & 20.0 & 2010-12-22.18 & 71.67 & $<5.1 \times 10^{-4}$ & $<1.9 \times 10^{36}$ \\
\hline XMM-Newton EPIC & 0650560201 & 18.7 & $2010-12-26.43$ & 75.92 & $(3.4 \pm 0.8) \times 10^{-3}$ & $(0.7 \pm 0.2) \times 10^{36}$ \\
\hline XMM-Newton EPIC & 0650560301 & 21.6 & 2011-01-04.76 & 85.25 & $(1.8 \pm 0.7) \times 10^{-3}$ & $(0.4 \pm 0.2) \times 10^{36}$ \\
\hline XMM-Newton EPIC & 0650560401 & 11.9 & 2011-01-15.01 & 95.50 & $(2.9 \pm 0.8) \times 10^{-3}$ & $(0.4 \pm 0.2) \times 10^{36}$ \\
\hline XMM-Newton EPIC & 0650560501 & 6.2 & 2011-01-25.30 & 105.79 & $<5.4 \times 10^{-3}$ & $<0.9 \times 10^{36}$ \\
\hline XMM-Newton EPIC & 0650560601 & 16.2 & 2011-02-04.00 & 115.49 & $(2.2 \pm 0.8) \times 10^{-3}$ & $(0.5 \pm 0.2) \times 10^{36}$ \\
\hline Chandra HRC-I & 13178 & 17.5 & 2011-02-17.15 & 128.64 & $<2.9 \times 10^{-4}$ & $<1.1 \times 10^{36}$ \\
\hline Chandra HRC-I & 13179 & 17.5 & 2011-02-27.25 & 138.74 & $<4.2 \times 10^{-4}$ & $<1.6 \times 10^{36}$ \\
\hline Chandra HRC-I & 13180 & 17.3 & $2011-03-10.12$ & 149.61 & $<6.4 \times 10^{-4}$ & $<2.4 \times 10^{36}$ \\
\hline
\end{tabular}

Notes. ${ }^{(a)}$ Telescope and instrument used for observation. ${ }^{(b)}$ Dead-time corrected exposure time of the observation. ${ }^{(c)}$ Start date of the observation. (d) Time in days after the outburst of nova M31N 2010-10f in the optical on 2010-10-11.51 (MJD = 55 480.51). ${ }^{(e)}$ Source count rates, X-ray luminosities (unabsorbed, black body fit, $0.2-10.0 \mathrm{keV}$ ) and upper limits were estimated according to Sect. 3. 
Table 2. Optical observations of Bol 126 with small telescopes.

\begin{tabular}{|c|c|c|c|c|c|}
\hline Observatory $^{a}$ & $\begin{array}{l}\text { Date } \\
\text { [UT] }\end{array}$ & $\begin{array}{l}\text { MJD } \\
\text { [d] }\end{array}$ & $\begin{array}{l}\Delta t^{b} \\
{[\mathrm{~d}]}\end{array}$ & $\begin{array}{c}\text { Mag. GC } \\
{[\mathrm{mag}]}\end{array}$ & $\begin{array}{l}\text { Mag. nova }{ }^{d} \\
{[\mathrm{mag}]}\end{array}$ \\
\hline Ondřejov & 2010-10-06T22:22:05 & 55475.93 & -4.58 & $16.72 \pm 0.02$ & - \\
\hline Super-LOTIS & 2010-10-07T03:02:50 & 55476.13 & -4.38 & $16.67 \pm 0.48$ & - \\
\hline Ondřejov & 2010-10-08T19:37:55 & 55477.82 & -2.69 & $16.69 \pm 0.02$ & - \\
\hline Super-LOTIS & 2010-10-09Т03:00:43 & 55478.13 & -2.38 & $16.53 \pm 0.16$ & - \\
\hline Miyaki-Argenteus & 2010-10-09Т14:19:17 & 55478.60 & -1.91 & $16.62 \pm 0.16$ & - \\
\hline Miyaki-Argenteus & 2010-10-10T15:01:01 & 55479.63 & -0.88 & $16.69 \pm 0.17$ & - \\
\hline Ondřejov & 2010-10-10T19:20:38 & 55479.81 & -0.70 & $16.70 \pm 0.02$ & - \\
\hline Super-LOTIS & 2010-10-11T02:12:57 & 55480.09 & -0.42 & $16.65 \pm 0.13$ & - \\
\hline Xingming & 2010-10-11T13:21:30 & 55480.56 & 0.05 & $16.31 \pm 0.22$ & $17.60 \pm 0.96$ \\
\hline Super-LOTIS & 2010-10-12T11:15:42 & 55481.47 & 0.96 & $15.62 \pm 0.14$ & $16.12 \pm 0.28$ \\
\hline Xingming & 2010-10-12T15:02:56 & 55481.63 & 1.12 & $15.67 \pm 0.26$ & $16.21 \pm 0.48$ \\
\hline Ondřejov & 2010-10-12T19:30:43 & 55481.81 & 1.30 & $15.90 \pm 0.02$ & $16.61 \pm 0.13$ \\
\hline Xingming & 2010-10-13Т13:24:07 & 55482.56 & 2.05 & $16.18 \pm 0.23$ & $17.23 \pm 0.77$ \\
\hline Ondřejov & 2010-10-13T20:39:50 & 55482.86 & 2.35 & $16.19 \pm 0.02$ & $17.26 \pm 0.22$ \\
\hline Xingming & 2010-10-14T14:01:17 & 55483.58 & 3.07 & $16.19 \pm 0.29$ & $17.25 \pm 0.94$ \\
\hline Miyaki-Argenteus & 2010-10-15T13:35:27 & 55484.57 & 4.06 & $16.30 \pm 0.19$ & $17.57 \pm 0.83$ \\
\hline Itagaki & 2010-10-16T09:22:11 & 55485.39 & 4.88 & $16.42 \pm 0.29$ & $18.04 \pm 1.62$ \\
\hline Xingming & 2010-10-16T13:35:54 & 55485.57 & 5.06 & $16.42 \pm 0.21$ & $18.03 \pm 1.26$ \\
\hline Miyaki-Argenteus & 2010-10-16T14:59:20 & 55485.62 & 5.11 & $16.48 \pm 0.15$ & $18.32 \pm 1.25$ \\
\hline Miyaki-Argenteus & 2010-10-17T12:57:21 & 55486.54 & 6.03 & $16.57 \pm 0.14$ & $18.94 \pm 2.04$ \\
\hline Xingming & 2010-10-17T15:32:06 & 55486.65 & 6.14 & $16.45 \pm 0.25$ & $18.19 \pm 1.65$ \\
\hline Itagaki & 2010-10-17T15:43:06 & 55486.65 & 6.14 & $16.49 \pm 0.21$ & $18.37 \pm 1.66$ \\
\hline Miyaki-Argenteus & 2010-10-18T12:05:45 & 55487.50 & 6.99 & $16.70 \pm 0.18$ & - \\
\hline Ondřejov & 2010-10-19T00:14:24 & 55488.01 & 7.50 & $16.54 \pm 0.02$ & $70 \pm$ \\
\hline Itagaki & 2010-10-22T08:47:15 & 55491.37 & 10.86 & $16.58 \pm 0.29$ & - \\
\hline Super-LOTIS & 2010-10-25T05:14:32 & 55494.22 & 13.71 & $16.47 \pm 0.14$ & - \\
\hline Miyaki-Argenteus & 2010-10-26T13:11:55 & 55495.55 & 15.04 & $16.48 \pm 0.15$ & - \\
\hline Super-LOTIS & 2010-10-27T03:45:12 & 55496.16 & 15.65 & $16.67 \pm 0.14$ & - \\
\hline Miyaki-Argenteus & 2010-10-27T15:24:36 & 55496.64 & 16.13 & $16.61 \pm 0.17$ & - \\
\hline Super-LOTIS & 2010-10-28T02:39:48 & 55497.11 & 16.60 & $16.70 \pm 0.14$ & - \\
\hline Ondřejov & 2010-10-28T16:52:19 & 55497.70 & 17.19 & $16.68 \pm 0.02$ & - \\
\hline Super-LOTIS & 2010-10-29T01:55:36 & 55498.08 & 17.57 & $16.64 \pm 0.13$ & - \\
\hline Miyaki-Argenteus & 2010-10-29T11:28:38 & 55498.48 & 17.97 & $16.93 \pm 0.19$ & - \\
\hline Ondřejov & 2010-10-29T17:03:50 & 55498.71 & 18.20 & $16.70 \pm 0.02$ & - \\
\hline Super-LOTIS & 2010-10-30T02:04:25 & 55499.09 & 18.58 & $16.67 \pm 0.12$ & - \\
\hline Super-LOTIS & 2010-11-01T05:35:20 & 55501.23 & 20.72 & $16.65 \pm 0.14$ & - \\
\hline Miyaki-Argenteus & 2010-11-01T12:35:39 & 55501.52 & 21.01 & $16.79 \pm 0.16$ & - \\
\hline Super-LOTIS & 2010-11-02Т02:35:30 & 55502.11 & 21.60 & $16.62 \pm 0.13$ & - \\
\hline Miyaki-Argenteus & 2010-11-02T13:43:47 & 55502.57 & 22.06 & $16.53 \pm 0.14$ & - \\
\hline Super-LOTIS & 2010-11-03Т02:34:53 & 55503.11 & 22.60 & $16.55 \pm 0.13$ & - \\
\hline Miyaki-Argenteus & 2010-11-03T12:01:05 & 55503.50 & 22.99 & $16.50 \pm 0.15$ & - \\
\hline Super-LOTIS & 2010-11-04T01:51:30 & 55504.08 & 23.57 & $16.57 \pm 0.12$ & - \\
\hline Miyaki-Argenteus & 2010-11-04T12:59:54 & 55504.54 & 24.03 & $16.68 \pm 0.13$ & - \\
\hline Super-LOTIS & 2010-11-05Т01:50:35 & 55505.08 & 24.57 & $16.63 \pm 0.13$ & - \\
\hline Miyaki-Argenteus & 2010-11-05T11:26:58 & 55505.48 & 24.97 & $16.68 \pm 0.14$ & - \\
\hline Super-LOTIS & 2010-11-09T02:31:25 & 55509.11 & 28.60 & $16.64 \pm 0.13$ & - \\
\hline
\end{tabular}

Notes. ${ }^{(a)}$ See Sect. 2.2 for a description of the telescopes used. ${ }^{(b)}$ Time in days after the optical nova outburst on MJD $=55480.32 .{ }^{(c)} R$ band magnitude of Bol 126, see also Fig. 2. ${ }^{(d)} R$ band magnitude of M31N 2010-10f assuming a quiescence magnitude for Bol 126 of $R=16.7$ mag, see also Fig. 5. 
Table 3. Optical PAndromeda observations of Bol 126.

\begin{tabular}{|c|c|c|c|c|}
\hline $\begin{array}{l}\text { Date } \\
\text { [UT] }\end{array}$ & $\begin{array}{l}\text { MJD } \\
{[\mathrm{d}]}\end{array}$ & $\begin{array}{l}\Delta t^{a} \\
{[\mathrm{~d}]}\end{array}$ & $\begin{array}{c}\text { Mag. GC }{ }^{b} \\
{[\mathrm{mag}]}\end{array}$ & Filter \\
\hline $2010-07-24.46$ & 55401.46 & -79.05 & $16.899 \pm 0.023$ & $r$ \\
\hline $2010-07-24.59$ & 55401.59 & -78.92 & $16.936 \pm 0.030$ & $r$ \\
\hline $2010-07-25.47$ & 55402.47 & -78.04 & $16.903 \pm 0.022$ & $r$ \\
\hline $2010-07-25.60$ & 55402.60 & -77.91 & $16.892 \pm 0.019$ & $r$ \\
\hline $2010-07-27.46$ & 55404.46 & -76.05 & $16.894 \pm 0.031$ & $r$ \\
\hline $2010-07-27.58$ & 55404.58 & -75.93 & $16.901 \pm 0.014$ & $r$ \\
\hline $2010-07-28.47$ & 55405.47 & -75.04 & $16.885 \pm 0.012$ & $r$ \\
\hline $2010-07-28.59$ & 55405.59 & -74.92 & $16.894 \pm 0.016$ & $r$ \\
\hline $2010-07-29.48$ & 55406.48 & -74.03 & $16.909 \pm 0.018$ & $r$ \\
\hline $2010-07-29.60$ & 55406.60 & -73.91 & $16.900 \pm 0.016$ & 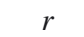 \\
\hline $2010-07-30.49$ & 55407.49 & -73.02 & $16.888 \pm 0.020$ & $r$ \\
\hline $2010-07-30.62$ & 55407.62 & -72.89 & $16.865 \pm 0.013$ & $r$ \\
\hline $2010-07-31.48$ & 55408.48 & -72.03 & $16.881 \pm 0.032$ & $r$ \\
\hline $2010-07-31.60$ & 55408.60 & -71.91 & $16.880 \pm 0.015$ & $r$ \\
\hline 2010-08-01.48 & 55409.48 & -71.03 & $16.892 \pm 0.016$ & $r$ \\
\hline 2010-08-01.60 & 55409.60 & -70.91 & $16.878 \pm 0.018$ & $r$ \\
\hline $2010-08-02.50$ & 55410.50 & -70.01 & $16.879 \pm 0.018$ & $r$ \\
\hline $2010-08-02.62$ & 55410.62 & -69.89 & $16.879 \pm 0.018$ & $r$ \\
\hline $2010-08-03.48$ & 55411.48 & -69.03 & $16.901 \pm 0.019$ & 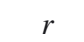 \\
\hline $2010-08-03.61$ & 55411.61 & -68.90 & $16.894 \pm 0.020$ & $r$ \\
\hline $2010-08-04.49$ & 55412.49 & -68.02 & $16.894 \pm 0.014$ & $r$ \\
\hline $2010-08-04.61$ & 55412.61 & -67.90 & $16.881 \pm 0.015$ & $r$ \\
\hline $2010-08-05.48$ & 55413.48 & -67.03 & $16.898 \pm 0.013$ & 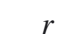 \\
\hline $2010-08-14.47$ & 55422.47 & -58.04 & $16.903 \pm 0.018$ & . \\
\hline $2010-08-14.62$ & 55422.62 & -57.89 & $16.901 \pm 0.020$ & $r$ \\
\hline $2010-08-15.49$ & 55423.49 & -57.02 & $16.895 \pm 0.018$ & $r$ \\
\hline $2010-08-15.62$ & 55423.62 & -56.89 & $16.886 \pm 0.019$ & $r$ \\
\hline $2010-08-16.49$ & 55424.49 & -56.02 & $16.892 \pm 0.012$ & $r$ \\
\hline $2010-08-16.62$ & 55424.62 & -55.89 & $16.878 \pm 0.017$ & $r$ \\
\hline 2010-08-17.49 & 55425.49 & -55.02 & $16.905 \pm 0.012$ & $r$ \\
\hline $2010-08-17.61$ & 55425.61 & -54.90 & $16.881 \pm 0.013$ & $r$ \\
\hline $2010-08-18.48$ & 55426.48 & -54.03 & $16.900 \pm 0.013$ & $r$ \\
\hline $2010-08-18.61$ & 55426.61 & -53.90 & $16.901 \pm 0.018$ & $r$ \\
\hline $2010-08-19.47$ & 55427.47 & -53.04 & $16.897 \pm 0.018$ & $r$ \\
\hline $2010-08-19.62$ & 55427.62 & -52.89 & $16.871 \pm 0.013$ & $r$ \\
\hline $2010-08-20.47$ & 55428.47 & -52.04 & $16.893 \pm 0.019$ & $r$ \\
\hline $2010-08-20.60$ & 55428.60 & -51.91 & $16.904 \pm 0.015$ & \\
\hline $2010-08-21.48$ & 55429.48 & -51.03 & $16.897 \pm 0.015$ & 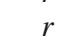 \\
\hline $2010-08-21.61$ & 55429.61 & -50.90 & $16.890 \pm 0.014$ & 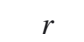 \\
\hline $2010-08-22.48$ & 55430.48 & -50.03 & $16.890 \pm 0.011$ & $r$ \\
\hline $2010-08-22.59$ & 55430.59 & -49.92 & $16.888 \pm 0.012$ & $r$ \\
\hline $2010-08-23.49$ & 55431.49 & -49.02 & $16.905 \pm 0.020$ & $r$ \\
\hline $2010-08-24.47$ & 55432.47 & -48.04 & $16.886 \pm 0.012$ & $r$ \\
\hline $2010-08-24.58$ & 55432.58 & -47.93 & $16.880 \pm 0.016$ & . \\
\hline $2010-08-25.45$ & 55433.45 & -47.06 & $16.906 \pm 0.017$ & $r$ \\
\hline $2010-08-25.59$ & 55433.59 & -46.92 & $16.878 \pm 0.023$ & $r$ \\
\hline $2010-08-26.48$ & 55434.48 & -46.03 & $16.885 \pm 0.015$ & $r$ \\
\hline $2010-08-26.63$ & 55434.63 & -45.88 & $16.905 \pm 0.016$ & \\
\hline $2010-08-27.48$ & 55435.48 & -45.03 & $16.895 \pm 0.023$ & \\
\hline $2010-08-27.60$ & 55435.60 & -44.91 & $16.895 \pm 0.016$ & $r$ \\
\hline $2010-08-28.48$ & 55436.48 & -44.03 & $16.887 \pm 0.016$ & 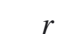 \\
\hline $2010-08-28.60$ & 55436.60 & -43.91 & $16.877 \pm 0.018$ & $r$ \\
\hline $2010-08-31.46$ & 55439.46 & -41.05 & $16.879 \pm 0.014$ & $r$ \\
\hline $2010-08-31.58$ & 55439.58 & -40.93 & $16.862 \pm 0.015$ & $r$ \\
\hline 2010-09-01.48 & 55440.48 & -40.03 & $16.861 \pm 0.012$ & $r$ \\
\hline 2010-09-01.62 & 55440.62 & -39.89 & $16.882 \pm 0.013$ & $r$ \\
\hline 2010-09-02.49 & 55441.49 & -39.02 & $16.863 \pm 0.013$ & $r$ \\
\hline 2010-09-02.61 & 55441.61 & -38.90 & $16.878 \pm 0.011$ & $r$ \\
\hline $2010-09-03.48$ & 55442.48 & -38.03 & $16.857 \pm 0.012$ & $r$ \\
\hline $2010-09-03.59$ & 55442.59 & -37.92 & $16.904 \pm 0.015$ & $r$ \\
\hline
\end{tabular}

Notes. ${ }^{(a)}$ Time in days after the optical nova outburst on MJD = $55480.51 .^{\left({ }^{(b)}\right.} r$ or $i$ band magnitude of Bol 126, see also Fig. 4.
Table 3. continued.

\begin{tabular}{|c|c|c|c|c|}
\hline $\begin{array}{l}\text { Date } \\
\text { [UT] }\end{array}$ & $\begin{array}{c}\text { MJD } \\
{[\mathrm{d}]}\end{array}$ & $\begin{array}{l}\Delta t^{a} \\
{[\mathrm{~d}]}\end{array}$ & $\begin{array}{c}\text { Mag. GC }{ }^{b} \\
{[\mathrm{mag}]}\end{array}$ & Filter \\
\hline 2010-09-04.45 & 55443.45 & -37.06 & $16.865 \pm 0.012$ & $r$ \\
\hline 2010-09-05.45 & 55444.45 & -36.06 & $16.884 \pm 0.012$ & $r$ \\
\hline 2010-09-05.57 & 55444.57 & -35.94 & $16.886 \pm 0.014$ & $r$ \\
\hline $2010-09-06.45$ & 55445.45 & -35.06 & $16.886 \pm 0.017$ & $r$ \\
\hline 2010-09-06.59 & 55445.59 & -34.92 & $16.901 \pm 0.014$ & $r$ \\
\hline $2010-09-07.44$ & 55446.44 & -34.07 & $16.890 \pm 0.014$ & $r$ \\
\hline $2010-09-07.58$ & 55446.58 & -33.93 & $16.886 \pm 0.013$ & $r$ \\
\hline 2010-09-08.44 & 55447.44 & -33.07 & $16.880 \pm 0.011$ & $r$ \\
\hline $2010-09-08.57$ & 55447.57 & -32.94 & $16.879 \pm 0.014$ & $r$ \\
\hline 2010-09-09.42 & 55448.42 & -32.09 & $16.894 \pm 0.016$ & $r$ \\
\hline $2010-09-09.54$ & 55448.54 & -31.97 & $16.892 \pm 0.019$ & 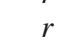 \\
\hline $2010-09-10.43$ & 55449.43 & -31.08 & $16.889 \pm 0.021$ & 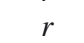 \\
\hline $2010-09-10.56$ & 55449.56 & -30.95 & $16.909 \pm 0.019$ & 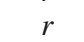 \\
\hline $2010-09-11.42$ & 55450.42 & -30.09 & $16.897 \pm 0.024$ & 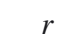 \\
\hline $2010-09-12.41$ & 55451.41 & -29.10 & $16.902 \pm 0.015$ & . \\
\hline $2010-09-12.55$ & 55451.55 & -28.96 & $16.888 \pm 0.015$ & $r$ \\
\hline $2010-09-13.42$ & 55452.42 & -28.09 & $16.881 \pm 0.014$ & $r$ \\
\hline $2010-09-13.56$ & 55452.56 & -27.95 & $16.897 \pm 0.018$ & 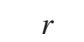 \\
\hline $2010-09-14.42$ & 55453.42 & -27.09 & $16.894 \pm 0.012$ & $r$ \\
\hline $2010-09-14.53$ & 55453.53 & -26.98 & $16.906 \pm 0.017$ & $r$ \\
\hline $2010-09-15.41$ & 55454.41 & -26.10 & $16.895 \pm 0.015$ & 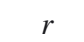 \\
\hline $2010-09-15.53$ & 55454.53 & -25.98 & $16.894 \pm 0.018$ & $r$ \\
\hline $2010-09-16.41$ & 55455.41 & -25.10 & $16.903 \pm 0.013$ & $r$ \\
\hline $2010-09-16.54$ & 55455.54 & -24.97 & $16.898 \pm 0.014$ & 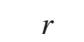 \\
\hline 2010-09-17.41 & 55456.41 & -24.10 & $16.901 \pm 0.013$ & 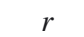 \\
\hline $2010-09-17.55$ & 55456.55 & -23.96 & $16.871 \pm 0.014$ & 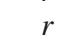 \\
\hline $2010-09-18.39$ & 55457.39 & -23.12 & $16.894 \pm 0.013$ & $r$ \\
\hline $2010-09-18.53$ & 55457.53 & -22.98 & $16.899 \pm 0.019$ & $r$ \\
\hline $2010-09-24.38$ & 55463.38 & -17.13 & $16.891 \pm 0.020$ & $r$ \\
\hline $2010-09-24.52$ & 55463.52 & -16.99 & $16.894 \pm 0.016$ & $r$ \\
\hline $2010-09-27.37$ & 55466.37 & -14.14 & $16.911 \pm 0.020$ & $r$ \\
\hline $2010-09-27.50$ & 55466.50 & -14.01 & $16.903 \pm 0.023$ & $r$ \\
\hline $2010-09-28.36$ & 55467.36 & -13.15 & $16.871 \pm 0.025$ & $r$ \\
\hline $2010-09-28.51$ & 55467.51 & -13.00 & $16.890 \pm 0.020$ & $r$ \\
\hline $2010-09-29.37$ & 55468.37 & -12.14 & $16.898 \pm 0.021$ & $r$ \\
\hline $2010-09-29.54$ & 55468.54 & -11.97 & $16.957 \pm 0.037$ & $r$ \\
\hline $2010-10-03.37$ & 55472.37 & -8.14 & $16.897 \pm 0.020$ & $r$ \\
\hline $2010-10-03.49$ & 55472.49 & -8.02 & $16.904 \pm 0.014$ & $r$ \\
\hline $2010-10-06.35$ & 55475.35 & -5.16 & $16.919 \pm 0.019$ & 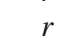 \\
\hline $2010-10-06.49$ & 55475.49 & -5.02 & $16.903 \pm 0.017$ & 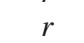 \\
\hline $2010-10-07.35$ & 55476.35 & -4.16 & $16.889 \pm 0.012$ & $r$ \\
\hline $2010-10-07.48$ & 55476.48 & -4.03 & $16.894 \pm 0.012$ & $r$ \\
\hline $2010-10-08.36$ & 55477.36 & -3.15 & $16.885 \pm 0.012$ & $r$ \\
\hline 2010-10-08.49 & 55477.49 & -3.02 & $16.892 \pm 0.013$ & $r$ \\
\hline $2010-10-09.35$ & 55478.35 & -2.16 & $16.896 \pm 0.011$ & $r$ \\
\hline $2010-10-09.49$ & 55478.49 & -2.02 & $16.906 \pm 0.014$ & $r$ \\
\hline $2010-10-10.34$ & 55479.34 & -1.17 & $16.905 \pm 0.017$ & $r$ \\
\hline $2010-10-10.48$ & 55479.48 & -1.03 & $16.928 \pm 0.017$ & $r$ \\
\hline $2010-10-11.34$ & 55480.34 & -0.17 & $16.903 \pm 0.020$ & $r$ \\
\hline $2010-10-11.46$ & 55480.46 & -0.05 & $16.880 \pm 0.016$ & $r$ \\
\hline $2010-10-12.34$ & 55481.34 & 0.83 & $15.512 \pm 0.009$ & $r$ \\
\hline $2010-10-12.46$ & 55481.46 & 0.95 & $15.839 \pm 0.014$ & $r$ \\
\hline $2010-10-13.51$ & 55482.51 & 2.00 & $16.220 \pm 0.009$ & 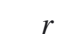 \\
\hline $2010-10-14.33$ & 55483.33 & 2.82 & $16.296 \pm 0.010$ & $r$ \\
\hline $2010-10-14.47$ & 55483.47 & 2.96 & $16.454 \pm 0.012$ & $r$ \\
\hline $2010-10-15.34$ & 55484.34 & 3.83 & $16.442 \pm 0.011$ & $r$ \\
\hline $2010-10-15.46$ & 55484.46 & 3.95 & $16.557 \pm 0.010$ & $r$ \\
\hline $2010-10-18.32$ & 55487.32 & 6.81 & $16.714 \pm 0.019$ & $r$ \\
\hline $2010-10-18.45$ & 55487.45 & 6.94 & $16.730 \pm 0.012$ & $r$ \\
\hline $2010-10-20.32$ & 55489.32 & 8.81 & $16.754 \pm 0.020$ & $r$ \\
\hline $2010-10-27.31$ & 55496.31 & 15.80 & $16.873 \pm 0.051$ & $r$ \\
\hline $2010-10-29.31$ & 55498.31 & 17.80 & $16.861 \pm 0.012$ & $r$ \\
\hline $2010-10-29.43$ & 55498.43 & 17.92 & $16.896 \pm 0.016$ & $r$ \\
\hline
\end{tabular}


Table 3. continued.

\begin{tabular}{|c|c|c|c|c|}
\hline $\begin{array}{l}\text { Date } \\
\text { [UT] }\end{array}$ & $\begin{array}{l}\text { MJD } \\
\text { [d] }\end{array}$ & $\begin{array}{l}\Delta t^{a} \\
{[\mathrm{~d}]}\end{array}$ & $\begin{array}{c}\text { Mag. GC }{ }^{b} \\
{[\mathrm{mag}]}\end{array}$ & Filter \\
\hline $2010-10-30.31$ & 55499.31 & 18.80 & $16.877 \pm 0.012$ & $r$ \\
\hline $2010-10-30.43$ & 55499.43 & 18.92 & $16.873 \pm 0.014$ & $r$ \\
\hline 2010-10-31.31 & 55500.31 & 19.80 & $16.866 \pm 0.011$ & $r$ \\
\hline $2010-10-31.44$ & 55500.44 & 19.93 & $16.872 \pm 0.011$ & $r$ \\
\hline $2010-11-01.27$ & 55501.27 & 20.76 & $16.879 \pm 0.013$ & $r$ \\
\hline 2010-11-01.43 & 55501.43 & 20.92 & $16.893 \pm 0.012$ & $r$ \\
\hline $2010-11-02.28$ & 55502.27 & 21.76 & $16.881 \pm 0.016$ & $r$ \\
\hline 2010-11-02.41 & 55502.41 & 21.90 & $16.882 \pm 0.010$ & $r$ \\
\hline 2010-11-03.27 & 55503.27 & 22.76 & $16.897 \pm 0.014$ & $r$ \\
\hline 2010-11-03.40 & 55503.40 & 22.89 & $16.880 \pm 0.012$ & $r$ \\
\hline 2010-11-07.29 & 55507.29 & 26.78 & $16.914 \pm 0.021$ & $r$ \\
\hline 2010-11-07.41 & 55507.41 & 26.90 & $16.889 \pm 0.011$ & $r$ \\
\hline 2010-11-08.28 & 55508.28 & 27.77 & $16.876 \pm 0.018$ & $r$ \\
\hline 2010-11-08.40 & 55508.40 & 27.89 & $16.885 \pm 0.013$ & $r$ \\
\hline 2010-11-10.27 & 55510.27 & 29.76 & $16.885 \pm 0.013$ & $r$ \\
\hline 2010-11-10.41 & 55510.41 & 29.90 & $16.896 \pm 0.014$ & $r$ \\
\hline 2010-11-11.28 & 55511.28 & 30.77 & $16.884 \pm 0.014$ & $r$ \\
\hline $2010-11-11.40$ & 55511.40 & 30.89 & $16.892 \pm 0.011$ & $r$ \\
\hline $2010-11-12.25$ & 55512.25 & 31.74 & $16.890 \pm 0.027$ & $r$ \\
\hline $2010-11-14.26$ & 55514.26 & 33.75 & $16.917 \pm 0.018$ & $r$ \\
\hline $2010-11-16.24$ & 55516.24 & 35.73 & $16.910 \pm 0.014$ & $r$ \\
\hline 2010-11-16.38 & 55516.38 & 35.86 & $16.893 \pm 0.011$ & $r$ \\
\hline $2010-11-18.25$ & 55518.25 & 37.74 & $16.896 \pm 0.013$ & $r$ \\
\hline 2010-11-18.39 & 55518.39 & 37.88 & $16.877 \pm 0.012$ & $r$ \\
\hline 2010-11-21.22 & 55521.22 & 40.71 & $16.897 \pm 0.016$ & $r$ \\
\hline 2010-11-21.39 & 55521.39 & 40.88 & $16.916 \pm 0.016$ & $r$ \\
\hline 2010-11-23.24 & 55523.24 & 42.73 & $16.920 \pm 0.013$ & $r$ \\
\hline 2010-11-23.37 & 55523.37 & 42.86 & $16.905 \pm 0.017$ & $r$ \\
\hline 2010-11-30.21 & 55530.21 & 49.70 & $16.887 \pm 0.030$ & $r$ \\
\hline 2010-11-30.36 & 55530.36 & 49.85 & $16.903 \pm 0.028$ & $r$ \\
\hline 2010-12-03.23 & 55533.23 & 52.72 & $16.913 \pm 0.174$ & $r$ \\
\hline 2010-12-04.23 & 55534.23 & 53.72 & $16.895 \pm 0.010$ & $r$ \\
\hline 2010-12-04.36 & 55534.36 & 53.85 & $16.894 \pm 0.012$ & $r$ \\
\hline $2010-12-05.23$ & 55535.23 & 54.72 & $16.909 \pm 0.017$ & $r$ \\
\hline 2010-12-05.35 & 55535.35 & 54.84 & $16.873 \pm 0.021$ & $r$ \\
\hline $2010-12-25.23$ & 55555.23 & 74.72 & $16.882 \pm 0.020$ & $r$ \\
\hline 2010-12-25.33 & 55555.33 & 74.82 & $16.893 \pm 0.023$ & $r$ \\
\hline $2010-12-27.22$ & 55557.22 & 76.71 & $16.883 \pm 0.020$ & $r$ \\
\hline $2010-12-27.33$ & 55557.33 & 76.82 & $16.907 \pm 0.018$ & $r$ \\
\hline $2011-07-25.61$ & 55767.61 & 287.10 & $16.960 \pm 0.015$ & $r$ \\
\hline 2011-07-26.61 & 55768.61 & 288.10 & $16.951 \pm 0.014$ & $r$ \\
\hline 2011-07-27.60 & 55769.60 & 289.09 & $16.957 \pm 0.007$ & $r$ \\
\hline $2011-07-28.59$ & 55770.59 & 290.08 & $16.952 \pm 0.013$ & $r$ \\
\hline 2011-07-31.53 & 55773.53 & 293.02 & $16.951 \pm 0.010$ & $r$ \\
\hline 2011-08-01.55 & 55774.55 & 294.04 & $16.946 \pm 0.008$ & $r$ \\
\hline 2011-08-02.56 & 55775.56 & 295.05 & $16.948 \pm 0.008$ & $r$ \\
\hline 2011-08-03.61 & 55776.61 & 296.10 & $16.963 \pm 0.008$ & $r$ \\
\hline 2011-08-04.47 & 55777.47 & 296.96 & $16.911 \pm 0.012$ & $r$ \\
\hline 2011-08-04.61 & 55777.61 & 297.10 & $16.961 \pm 0.012$ & $r$ \\
\hline 2011-08-06.48 & 55779.48 & 298.97 & $16.942 \pm 0.010$ & $r$ \\
\hline 2011-08-06.61 & 55779.61 & 299.10 & $16.959 \pm 0.008$ & $r$ \\
\hline 2011-08-09.48 & 55782.48 & 301.97 & $16.916 \pm 0.024$ & $r$ \\
\hline 2011-08-09.61 & 55782.61 & 302.10 & $16.939 \pm 0.014$ & $r$ \\
\hline 2011-08-10.47 & 55783.47 & 302.96 & $16.943 \pm 0.010$ & 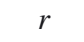 \\
\hline 2011-08-10.62 & 55783.62 & 303.11 & $16.913 \pm 0.008$ & r \\
\hline 2011-08-11.48 & 55784.48 & 303.97 & $16.947 \pm 0.011$ & $r$ \\
\hline 2011-08-11.59 & 55784.59 & 304.08 & $16.895 \pm 0.009$ & $r$ \\
\hline 2011-08-12.48 & 55785.48 & 304.97 & $16.958 \pm 0.009$ & $r$ \\
\hline 2010-07-23.53 & 55400.53 & -79.98 & $16.713 \pm 0.016$ & $i$ \\
\hline $2010-07-24.45$ & 55401.45 & -79.06 & $16.702 \pm 0.022$ & $i$ \\
\hline 2010-07-24.58 & 55401.58 & -78.93 & $16.690 \pm 0.027$ & $i$ \\
\hline $2010-07-25.47$ & 55402.47 & -78.04 & $16.702 \pm 0.021$ & $i$ \\
\hline $2010-07-25.60$ & 55402.60 & -77.91 & $16.684 \pm 0.016$ & $i$ \\
\hline
\end{tabular}

Table 3. continued.

\begin{tabular}{|c|c|c|c|c|}
\hline $\begin{array}{l}\text { Date } \\
\text { [UT] }\end{array}$ & $\begin{array}{l}\text { MJD } \\
{[\mathrm{d}]}\end{array}$ & $\begin{array}{l}\Delta t^{a} \\
{[\mathrm{~d}]}\end{array}$ & $\begin{array}{c}\text { Mag. GC } \\
\text { [mag] }\end{array}$ & Filter \\
\hline $2010-07-27.46$ & 55404.46 & -76.05 & $16.694 \pm 0.038$ & $\bar{i}$ \\
\hline $2010-07-27.58$ & 55404.58 & -75.93 & $16.695 \pm 0.011$ & $i$ \\
\hline $2010-07-28.46$ & 55405.46 & -75.05 & $16.693 \pm 0.011$ & $i$ \\
\hline 2010-07-28.58 & 55405.58 & -74.93 & $16.692 \pm 0.011$ & $i$ \\
\hline $2010-07-29.47$ & 55406.47 & -74.04 & $16.704 \pm 0.019$ & $i$ \\
\hline $2010-07-29.59$ & 55406.59 & -73.92 & $16.677 \pm 0.012$ & $i$ \\
\hline $2010-07-30.49$ & 55407.49 & -73.02 & $16.693 \pm 0.013$ & $i$ \\
\hline $2010-07-30.61$ & 55407.61 & -72.90 & $16.669 \pm 0.012$ & $i$ \\
\hline $2010-07-31.47$ & 55408.47 & -72.04 & $16.702 \pm 0.029$ & $i$ \\
\hline $2010-07-31.60$ & 55408.60 & -71.91 & $16.697 \pm 0.012$ & $i$ \\
\hline 2010-08-01.48 & 55409.48 & -71.03 & $16.685 \pm 0.013$ & $i$ \\
\hline $2010-08-01.59$ & 55409.59 & -70.92 & $16.672 \pm 0.013$ & $i$ \\
\hline $2010-08-02.50$ & 55410.50 & -70.01 & $16.693 \pm 0.017$ & $i$ \\
\hline $2010-08-02.62$ & 55410.62 & -69.89 & $16.722 \pm 0.024$ & $i$ \\
\hline $2010-08-03.48$ & 55411.48 & -69.03 & $16.710 \pm 0.014$ & $i$ \\
\hline $2010-08-03.60$ & 55411.60 & -68.91 & $16.676 \pm 0.014$ & $i$ \\
\hline 2010-08-04.48 & 55412.48 & -68.03 & $16.700 \pm 0.012$ & $i$ \\
\hline $2010-08-04.60$ & 55412.60 & -67.91 & $16.678 \pm 0.011$ & $i$ \\
\hline $2010-08-05.47$ & 55413.47 & -67.04 & $16.695 \pm 0.011$ & $i$ \\
\hline $2010-08-05.61$ & 55413.61 & -66.90 & $16.692 \pm 0.014$ & $i$ \\
\hline $2010-08-22.47$ & 55430.47 & -50.04 & $16.696 \pm 0.009$ & $i$ \\
\hline $2010-08-22.58$ & 55430.58 & -49.93 & $16.681 \pm 0.012$ & $i$ \\
\hline $2010-08-23.49$ & 55431.49 & -49.02 & $16.703 \pm 0.012$ & $i$ \\
\hline $2010-08-24.46$ & 55432.46 & -48.05 & $16.691 \pm 0.010$ & $i$ \\
\hline $2010-08-24.57$ & 55432.57 & -47.94 & $16.680 \pm 0.019$ & $i$ \\
\hline $2010-08-25.45$ & 55433.45 & -47.06 & $16.718 \pm 0.016$ & $i$ \\
\hline $2010-08-25.58$ & 55433.58 & -46.93 & $16.685 \pm 0.011$ & $i$ \\
\hline $2010-08-26.48$ & 55434.48 & -46.03 & $16.692 \pm 0.012$ & $i$ \\
\hline $2010-08-26.61$ & 55434.61 & -45.90 & $16.670 \pm 0.018$ & $i$ \\
\hline $2010-08-27.47$ & 55435.47 & -45.04 & $16.689 \pm 0.015$ & $i$ \\
\hline $2010-08-27.59$ & 55435.59 & -44.92 & $16.684 \pm 0.013$ & $i$ \\
\hline $2010-08-28.47$ & 55436.47 & -44.04 & $16.684 \pm 0.015$ & $i$ \\
\hline $2010-08-28.60$ & 55436.60 & -43.91 & $16.671 \pm 0.011$ & $i$ \\
\hline $2010-08-31.46$ & 55439.46 & -41.05 & $16.685 \pm 0.012$ & $i$ \\
\hline $2010-08-31.59$ & 55439.59 & -40.92 & $16.683 \pm 0.011$ & $i$ \\
\hline 2010-09-01.48 & 55440.48 & -40.03 & $16.688 \pm 0.011$ & $i$ \\
\hline 2010-09-01.61 & 55440.61 & -39.90 & $16.692 \pm 0.011$ & $i$ \\
\hline $2010-09-02.48$ & 55441.48 & -39.03 & $16.668 \pm 0.012$ & $i$ \\
\hline 2010-09-02.60 & 55441.60 & -38.91 & $16.662 \pm 0.010$ & $i$ \\
\hline $2010-09-03.47$ & 55442.47 & -38.04 & $16.676 \pm 0.010$ & $i$ \\
\hline 2010-09-03.59 & 55442.59 & -37.92 & $16.675 \pm 0.012$ & $i$ \\
\hline $2010-09-04.45$ & 55443.45 & -37.06 & $16.690 \pm 0.010$ & $i$ \\
\hline 2010-09-05.45 & 55444.45 & -36.06 & $16.690 \pm 0.011$ & $i$ \\
\hline 2010-09-05.57 & 55444.57 & -35.94 & $16.684 \pm 0.011$ & $i$ \\
\hline 2010-09-06.45 & 55445.45 & -35.06 & $16.682 \pm 0.012$ & $i$ \\
\hline 2010-09-06.58 & 55445.58 & -34.93 & $16.689 \pm 0.012$ & $i$ \\
\hline 2010-09-07.44 & 55446.44 & -34.07 & $16.689 \pm 0.011$ & $i$ \\
\hline $2010-09-07.58$ & 55446.58 & -33.93 & $16.684 \pm 0.012$ & $i$ \\
\hline $2010-09-28.37$ & 55467.37 & -13.14 & $16.693 \pm 0.012$ & $i$ \\
\hline $2010-09-28.51$ & 55467.51 & -13.00 & $16.659 \pm 0.026$ & $i$ \\
\hline $2010-09-29.37$ & 55468.37 & -12.14 & $16.685 \pm 0.024$ & $i$ \\
\hline $2010-09-29.55$ & 55468.55 & -11.96 & $16.685 \pm 0.027$ & $i$ \\
\hline $2010-10-03.37$ & 55472.37 & -8.14 & $16.700 \pm 0.019$ & $i$ \\
\hline $2010-10-03.50$ & 55472.50 & -8.01 & $16.694 \pm 0.012$ & $i$ \\
\hline $2010-10-06.35$ & 55475.35 & -5.16 & $16.706 \pm 0.016$ & $i$ \\
\hline $2010-10-06.48$ & 55475.48 & -5.03 & $16.703 \pm 0.013$ & $i$ \\
\hline 2010-10-09.35 & 55478.35 & -2.16 & $16.692 \pm 0.011$ & $i$ \\
\hline $2010-10-09.50$ & 55478.50 & -2.01 & $16.690 \pm 0.011$ & $i$ \\
\hline $2010-10-10.35$ & 55479.35 & -1.16 & $16.706 \pm 0.015$ & $i$ \\
\hline $2010-10-10.49$ & 55479.49 & -1.02 & $16.692 \pm 0.016$ & $i$ \\
\hline $2010-10-11.35$ & 55480.35 & -0.16 & $16.699 \pm 0.018$ & $i$ \\
\hline $2010-10-11.46$ & 55480.46 & -0.05 & $16.684 \pm 0.015$ & $i$ \\
\hline $2010-10-12.35$ & 55481.35 & 0.84 & $15.602 \pm 0.009$ & $i$ \\
\hline
\end{tabular}


M. Henze et al.: X-rays reveal second M 31 globular cluster nova

Table 3. continued.

\begin{tabular}{|c|c|c|c|c|}
\hline $\begin{array}{l}\text { Date } \\
\text { [UT] }\end{array}$ & $\begin{array}{l}\text { MJD } \\
{[\mathrm{d}]}\end{array}$ & $\begin{array}{l}\Delta t^{a} \\
{[\mathrm{~d}]} \\
\end{array}$ & $\begin{array}{c}\text { Mag. } \mathrm{GC}^{b} \\
{[\mathrm{mag}]}\end{array}$ & Filter \\
\hline $2010-10-12.46$ & 55481.46 & 0.95 & $15.702 \pm 0.010$ & $i$ \\
\hline $2010-10-13.33$ & 55482.33 & 1.82 & $16.118 \pm 0.032$ & $i$ \\
\hline $2010-10-13.50$ & 55482.50 & 1.99 & $16.169 \pm 0.009$ & $i$ \\
\hline $2010-10-14.32$ & 55483.32 & 2.81 & $16.349 \pm 0.011$ & $i$ \\
\hline $2010-10-14.46$ & 55483.46 & 2.95 & $16.415 \pm 0.010$ & $i$ \\
\hline $2010-10-15.33$ & 55484.33 & 3.82 & $16.491 \pm 0.010$ & $i$ \\
\hline $2010-10-15.46$ & 55484.46 & 3.95 & $16.525 \pm 0.010$ & $i$ \\
\hline $2010-10-18.33$ & 55487.33 & 6.82 & $16.651 \pm 0.020$ & $i$ \\
\hline $2010-10-18.45$ & 55487.45 & 6.94 & $16.635 \pm 0.011$ & $i$ \\
\hline $2010-10-20.31$ & 55489.31 & 8.80 & $16.660 \pm 0.024$ & $i$ \\
\hline $2010-10-27.30$ & 55496.30 & 15.79 & $16.610 \pm 0.055$ & $i$ \\
\hline $2010-10-29.30$ & 55498.30 & 17.79 & $16.688 \pm 0.010$ & $i$ \\
\hline $2010-10-29.43$ & 55498.43 & 17.92 & $16.667 \pm 0.016$ & $i$ \\
\hline $2010-10-30.30$ & 55499.30 & 18.79 & $16.693 \pm 0.013$ & $i$ \\
\hline $2010-10-30.43$ & 55499.43 & 18.92 & $16.673 \pm 0.012$ & $i$ \\
\hline $2010-10-31.31$ & 55500.31 & 19.80 & $16.687 \pm 0.011$ & $i$ \\
\hline $2010-10-31.43$ & 55500.43 & 19.92 & $16.674 \pm 0.012$ & $i$ \\
\hline 2010-11-01.28 & 55501.28 & 20.77 & $16.697 \pm 0.012$ & $i$ \\
\hline $2010-11-01.43$ & 55501.43 & 20.92 & $16.688 \pm 0.012$ & $i$ \\
\hline $2010-11-02.28$ & 55502.28 & 21.77 & $16.699 \pm 0.012$ & $i$ \\
\hline $2010-11-02.42$ & 55502.42 & 21.91 & $16.685 \pm 0.010$ & $i$ \\
\hline $2010-11-03.28$ & 55503.28 & 22.77 & $16.697 \pm 0.013$ & $i$ \\
\hline 2010-11-03.41 & 55503.41 & 22.90 & $16.678 \pm 0.012$ & $i$ \\
\hline $2010-11-07.28$ & 55507.28 & 26.77 & $16.691 \pm 0.017$ & $i$ \\
\hline $2010-11-07.40$ & 55507.40 & 26.89 & $16.688 \pm 0.010$ & $i$ \\
\hline $2010-11-08.27$ & 55508.27 & 27.76 & $16.695 \pm 0.014$ & $i$ \\
\hline $2010-11-08.39$ & 55508.39 & 27.88 & $16.692 \pm 0.014$ & $i$ \\
\hline $2010-11-10.27$ & 55510.27 & 29.76 & $16.689 \pm 0.012$ & $i$ \\
\hline $2010-11-10.40$ & 55510.40 & 29.89 & $16.689 \pm 0.014$ & $i$ \\
\hline $2010-11-11.27$ & 55511.27 & 30.76 & $16.702 \pm 0.014$ & $i$ \\
\hline $2010-11-11.40$ & 55511.40 & 30.89 & $16.699 \pm 0.011$ & $i$ \\
\hline $2010-11-12.25$ & 55512.25 & 31.74 & $16.714 \pm 0.017$ & $i$ \\
\hline $2010-11-14.27$ & 55514.27 & 33.76 & $16.710 \pm 0.014$ & $i$ \\
\hline $2010-11-16.25$ & 55516.25 & 35.74 & $16.706 \pm 0.012$ & $i$ \\
\hline $2010-11-16.38$ & 55516.38 & 35.87 & $16.685 \pm 0.010$ & $i$ \\
\hline $2010-11-18.25$ & 55518.25 & 37.74 & $16.707 \pm 0.010$ & $i$ \\
\hline $2010-11-18.38$ & 55518.38 & 37.87 & $16.684 \pm 0.011$ & $i$ \\
\hline $2010-11-21.22$ & 55521.22 & 40.71 & $16.711 \pm 0.013$ & $i$ \\
\hline $2010-11-23.23$ & 55523.23 & 42.72 & $16.697 \pm 0.013$ & $i$ \\
\hline $2010-11-23.36$ & 55523.36 & 42.85 & $16.686 \pm 0.013$ & $i$ \\
\hline $2010-11-30.21$ & 55530.21 & 49.70 & $16.701 \pm 0.019$ & $i$ \\
\hline $2010-11-30.35$ & 55530.35 & 49.84 & $16.659 \pm 0.025$ & $i$ \\
\hline $2010-12-03.22$ & 55533.22 & 52.71 & $16.662 \pm 0.046$ & $i$ \\
\hline $2010-12-04.22$ & 55534.22 & 53.71 & $16.691 \pm 0.011$ & $i$ \\
\hline $2010-12-04.35$ & 55534.35 & 53.84 & $16.688 \pm 0.011$ & $i$ \\
\hline $2010-12-05.22$ & 55535.22 & 54.71 & $16.690 \pm 0.016$ & $i$ \\
\hline $2010-12-05.35$ & 55535.35 & 54.84 & $16.677 \pm 0.017$ & $i$ \\
\hline $2010-12-06.23$ & 55536.23 & 55.72 & $16.691 \pm 0.016$ & $i$ \\
\hline 2010-12-06.35 & 55536.35 & 55.84 & $16.666 \pm 0.029$ & $i$ \\
\hline $2010-12-25.22$ & 55555.22 & 74.71 & $16.675 \pm 0.020$ & $i$ \\
\hline $2010-12-25.33$ & 55555.33 & 74.82 & $16.662 \pm 0.022$ & $i$ \\
\hline $2010-12-27.21$ & 55557.21 & 76.70 & $16.673 \pm 0.016$ & $i$ \\
\hline $2010-12-27.32$ & 55557.32 & 76.81 & $16.686 \pm 0.017$ & $i$ \\
\hline 2011-07-25.61 & 55767.61 & 287.10 & $16.717 \pm 0.015$ & $i$ \\
\hline $2011-07-26.62$ & 55768.62 & 288.11 & $16.727 \pm 0.012$ & $i$ \\
\hline $2011-07-27.60$ & 55769.60 & 289.09 & $16.721 \pm 0.013$ & $i$ \\
\hline 2011-07-28.59 & 55770.59 & 290.08 & $16.735 \pm 0.018$ & $i$ \\
\hline $2011-07-31.53$ & 55773.53 & 293.02 & $16.729 \pm 0.018$ & $i$ \\
\hline $2011-08-01.55$ & 55774.55 & 294.04 & $16.725 \pm 0.011$ & $i$ \\
\hline $2011-08-02.56$ & 55775.56 & 295.05 & $16.714 \pm 0.010$ & $i$ \\
\hline 2011-08-03.61 & 55776.61 & 296.10 & $16.727 \pm 0.010$ & $i$ \\
\hline 2011-08-04.48 & 55777.48 & 296.97 & $16.677 \pm 0.015$ & $i$ \\
\hline 2011-08-04.61 & 55777.61 & 297.10 & $16.779 \pm 0.019$ & $i$ \\
\hline
\end{tabular}

Table 3. continued.

\begin{tabular}{lcccc}
\hline \hline $\begin{array}{l}\text { Date } \\
{[\mathrm{UT}]}\end{array}$ & $\begin{array}{c}\text { MJD } \\
{[\mathrm{d}]}\end{array}$ & $\begin{array}{c}\Delta t^{a} \\
{[\mathrm{~d}]}\end{array}$ & $\begin{array}{c}\text { Mag. GC } \\
{[\mathrm{mag}]}\end{array}$ & Filter \\
\hline $2011-08-06.48$ & 55779.48 & 298.97 & $16.696 \pm 0.011$ & $i$ \\
$2011-08-06.62$ & 55779.62 & 299.11 & $16.710 \pm 0.011$ & $i$ \\
$2011-08-09.48$ & 55782.48 & 301.97 & $16.693 \pm 0.020$ & $i$ \\
$2011-08-09.62$ & 55782.62 & 302.11 & $16.742 \pm 0.014$ & $i$ \\
$2011-08-10.46$ & 55783.46 & 302.95 & $16.685 \pm 0.010$ & $i$ \\
$2011-08-10.62$ & 55783.62 & 303.11 & $16.724 \pm 0.009$ & $i$ \\
$2011-08-11.48$ & 55784.48 & 303.97 & $16.713 \pm 0.012$ & $i$ \\
$2011-08-11.58$ & 55784.58 & 304.07 & $16.696 \pm 0.012$ & $i$ \\
$2011-08-12.49$ & 55785.49 & 304.98 & $16.702 \pm 0.020$ & $i$ \\
\hline
\end{tabular}

